\title{
Neurochemically and Hodologically Distinct Ascending VGLUT3 versus Serotonin Subsystems Comprise the r2-Pet1 Median Raphe
}

\author{
${ }^{\circledR}$ Rebecca A. Senft, ${ }^{\circledR}$ Morgan E. Freret, ${ }^{\circledR}$ Nikita Sturrock, and ${ }^{\circledR}$ Susan M. Dymecki \\ Department of Genetics, Blavatnik Institute, Harvard Medical School, Boston, Massachusetts 02115
}

Brainstem median raphe (MR) neurons expressing the serotonergic regulator gene Pet1 send collateralized projections to forebrain regions to modulate affective, memory-related, and circadian behaviors. Some Pet1 neurons express a surprisingly incomplete battery of serotonin pathway genes, with somata lacking transcripts for tryptophan hydroxylase 2 (Tph2) encoding the rate-limiting enzyme for serotonin [5-hydroxytryptamine (5-HT)] synthesis, but abundant for vesicular glutamate transporter type 3 (Vglut3) encoding a synaptic vesicle-associated glutamate transporter. Genetic fate maps show these nonclassical, putatively glutamatergic Pet1 neurons in the MR arise embryonically from the same progenitor cell compartment-hindbrain rhombomere $2(\mathrm{r} 2)$-as serotonergic $\mathrm{TPH}_{2}{ }^{+}$MR Pet1 neurons. Well established is the distribution of efferents en masse from r2-derived, Pet1-neurons; unknown is the relationship between these efferent targets and the specific constituent source-neuron subgroups identified as $\mathrm{r}^{2-P e t 1^{\text {Tph2-high }}}$ versus $\mathrm{r} 2$-Pet $1^{\text {Vglut3-high }}$. Using male and female mice, we found r2-Pet1 axonal boutons segregated anatomically largely by serotonin ${ }^{+}$versus VGLUT3 ${ }^{+}$identity. The former present in the suprachiasmatic nucleus, paraventricular nucleus of the thalamus, and olfactory bulb; the latter are found in the hippocampus, cortex,

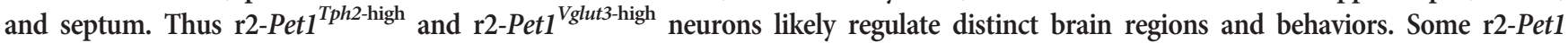
boutons encased interneuron somata, forming specialized presynaptic "baskets" of VGLUT3 ${ }^{+}$or VGLUT3 ${ }^{+} / 5-\mathrm{HT}^{+}$identity; this suggests that some $\mathrm{r} 2$-Pet1 ${ }^{\mathrm{Vglut} 3 \text {-high }}$ neurons may regulate local networks, perhaps with differential kinetics via glutamate versus serotonin signaling. Fibers from other Pet1 neurons (non-r2-derived) were observed in many of these same baskets, suggesting multifaceted regulation. Collectively, these findings inform brain organization and new circuit nodes for therapeutic considerations.

Key words: median raphe; neuronal subsystems; pericellular basket; Pet1; serotonin; VGLUT3

\section{Significance Statement}

Our findings match axonal bouton neurochemical identity with distant cell bodies in the brainstem raphe. The results are significant because they suggest that disparate neuronal subsystems derive from $\mathrm{Pet1}^{+}$precursor cells of the embryonic progenitor compartment rhombomere 2 (r2). Of these r2-Pet1 neuronal subsystems, one appears largely serotonergic, as expected given expression of the serotonergic regulator PET1, and projects to the olfactory bulb, thalamus, and suprachiasmatic nucleus. Another expresses VGLUT3, suggesting principally glutamate transmission, and projects to the hippocampus, septum, and cortex. Some r2-Pet1 boutons-those that are VGLUT3 ${ }^{+}$or VGLUT3 ${ }^{+} / 5-\mathrm{HT}^{+}$co-positive-comprise "baskets" encasing interneurons, suggesting that they control local networks perhaps with differential kinetics via glutamate versus serotonin signaling. Results inform brain organization and circuit nodes for therapeutic consideration.

Received June 29, 2020; revised Jan. 1, 2021; accepted Jan. 26, 2021.

Author contributions: R.A.S., M.E.F., N.S., and S.M.D. designed research; R.A.S., M.E.F., and N.S. performed research; R.A.S. and M.E.F. analyzed data; R.A.S., M.E.F., and S.M.D. wrote the paper.

This work was supported by National Institutes of Health Grants F31-NS-108406 (to R.A.S.), T32-HL-007901 (to N.S.), and R21-DA-036056 (to S.M.D. and M.E.F.). The experiments presented in this article were made possible by the use of microscopes in the MicRoN Microscopy Core (Harvard Medical School), use of Imaris software in the Neurobiology Imaging Facility (Harvard Medical School; funded by National Institute of Neurological Disorders and Stroke P30 Core Center Grant NS-072030) and critical conversations with Dr. Paula Montero-Llopis and Ryan Stephansky of MicRoN. We thank Drs. Plummer, Jensen, and colleagues for sharing the RC-FLTG mouse line. We also thank Drs. Benjamin Okaty, Ryan Dosumu-Johnson, and Benjamin Rood for discussions and feedback; and Jia Jia Mai for technical support.

The authors declare no competing financial interests.

Correspondence should be addressed to Susan M. Dymecki at dymecki@genetics.med.harvard.edu. https://doi.org/10.1523/JNEUROSCI.1667-20.2021

Copyright $\odot 2021$ the authors

\section{Introduction}

Neurons of the brainstem median raphe (MR) that express the serotonergic regulator gene Pet1 (alias Fev) project to numerous forebrain sites (Kosofsky and Molliver, 1987; Kiyasova and Gaspar, 2011; Bang et al., 2012; Gaspar and Lillesaar, 2012) to modulate diverse processes including emotional behavior (Teissier et al., 2015; Abela et al., 2020), sensorimotor gating (Okaty et al., 2015), memory (Ohmura et al., 2010; Baskin et al., 2020), and sleep and circadian rhythms (Meyer-Bernstein and Morin, 1996; Glass et al., 2003; Iwasaki et al., 2018). Recent work shows that some of these MR Pet1 neurons, even with their 
expression of Pet1, lack detectable transcript levels for various serotonin pathway genes (Pelosi et al., 2014; Okaty et al., 2015, 2019; Sos et al., 2017; Ren et al., 2019), but demonstrate high levels of Slc17a8 (alias Vglut3) mRNA, which encodes the type 3 transporter [vesicular glutamate transporter type 3 (VGLUT3)] for loading glutamate into synaptic vesicles. Genetic fate maps coupled with single-cell transcriptomic analyses show that these $\operatorname{Vglut}^{+}$, tryptophan hydroxylase $2(\mathrm{Tph} 2)^{-}$, putative glutamatergic MR Pet 1 neurons arise embryonically from the same progenitor cell compartment-hindbrain rhombomere 2 (r2)-as do more classically serotonergic $T p h 2^{+}$, serotonin [5-hydroxytryptamine (5-HT) ${ }^{+}$] MR Pet1 neurons (Okaty et al., 2015). Though the brain regions innervated by the collective r2-derived Pet 1 neuron population have been mapped (Bang et al., 2012), the degree to which the nonclassical r2-Pet1 $1^{\text {Vglut } 3 \text {-high }}$ neuron subgroup and the classical $\mathrm{r} 2-P e t 1^{\text {Tph2-high }}$ neuron subgroup differentially contribute to these projections is unknown. Here we present progress toward resolving this circuitry.

One possibility is that the $\mathrm{r} 2-\mathrm{Pet1}^{\text {Tph2-high }}$ and $\mathrm{r} 2-\mathrm{Pet}^{\text {Vglut3-high }}$ neuron subgroups exhibit converging circuitry, innervating the same target regions and potentially exerting regulatory effects of different directions and/or timescales. An alternative extreme is that they exhibit divergent circuitry, each innervating different brain regions to control different functions. Also possible is a combination of both as a function of target site. Because neurochemical identity (serotonin and TPH2 vs VGLUT3 as a proxy for glutamate) largely distinguishes the $\mathrm{r} 2-$ Pet $^{\text {Tph2-high }}$ from $\mathrm{r} 2$ Pet $1^{\text {Vglut3-high }}$ neuron somata, we reasoned it might also distinguish their boutons and thus target brain regions, which in turn could help discern from among these circuitry possibilities.

In this study, we used dual recombinase-based genetic labeling strategies (Awatramani et al., 2003; Bang et al., 2012), retrograde viral tracing, immunohistology, and single-cell in situ mRNA detection combined with confocal microscopy to describe the neurochemical identity and distribution of axonal boutons arising from the r2-Pet1 neuron population. We found anatomic segregation of $\mathrm{r} 2-\mathrm{Pet} 1$ axonal boutons by serotonin ${ }^{+}$ versus $\mathrm{VGLUT3}^{+}$identity, suggesting that the r2-Pet $^{\text {Tph2-high }}$ and $\mathrm{r} 2$-Pet $1^{\text {Vglut3-high }}$ neuron subgroups innervate distinct brain regions. Further, we found that some VGLUT3 ${ }^{+}$r2-Pet1 boutons comprised a specialized presynaptic structure that has been called a pericellular basket (Köhler et al., 1982; Hornung and Celio, 1992; Dinopoulos et al., 1993; Riedel et al., 2008), here found encasing the soma and dendrites of individual hippocampal, cortical, and septal GABAergic neurons of specific subclasses. This finding suggests that some r2-Pet1 $1^{\text {Vglut3-high }}$ neurons may have a privileged capacity to control local network excitability. In the septum, we observed co-positive VGLUT3 ${ }^{+} / 5-\mathrm{HT}^{+}$ r2-Pet1 boutons, and they were enriched in these pericellular baskets, suggesting that this may be a specialized site of 5-HT/ glutamate cotransmission. Further, we observed that the septal cells targeted by $\mathrm{r} 2$-Pet 1 baskets commonly also received serotonin $^{+}$fibers from other (non-r2) Pet1 neurons, suggesting even additional regulatory complexity. This work provides a foundational framework for pursuit of downstream circuits and cells relevant to specific behavioral modulation by r2-Pet1 neurons, and further extends our knowledge of the functional organization of the median raphe nucleus.

\section{Materials and Methods}

Animals

All experimental procedures were approved by the Harvard Institutional Animal Care and Use Committee. Mice were housed in a colony maintained on a $12 \mathrm{~h}$ light/dark cycle. All transgenic strains described below have been previously backcrossed to the C57BL/6J inbred strain (stock \#000664, The Jackson Laboratory) for at least nine generations. In all experiments, adult mice were used [at least postnatal day 60 (P60)], as previous research has shown that the postnatal maturation of serotonin neuron fiber morphology is finalized before this age (Maddaloni et al., 2017).

For projection mapping studies, r2-Pet1 neuron boutons were visualized by cell-autonomous expression of a synaptophysin-GFP (synGFP) fusion protein using intersectional genetics. These studies used tripletransgenic mice harboring the r2-specific Cre driver Hoxa2-cre (alias r2Hoxa2-cre; Awatramani et al., 2003) and the Flp driver Pet1-Flpe (Jensen et al., 2008) in combination with the dual Cre/Flp-responsive allele Gt(ROSA)26Sor tm10(CAG-Syp/EGFP+,-tdTomato)Dym (termed RC-FPSit; stock \#030206, The Jackson Laboratory; Niederkofler et al., 2016). Triple-transgenic 2 Hoxa2-cre, Pet1-Flpe, RC-FPSit ${ }^{+/-}$mice were generated by crossing double-transgenic Pet1-Flpe, RC-FPSit ${ }^{-1-}$ males to r2Hoxa2-cre females. To fluorescently label the somata and fibers of r2-Pet1 neurons with EGFP (called the intersectional cell population) and all other Pet 1 neurons with mCherry (called the subtractive cell population), we generated triple-transgenic mice bearing the drivers r2Hoxa2-cre and Pet1-Flpe, together with the dual Cre/ Flp-responsive ROSA26 allele Gt(ROSA)26Sor tm8(CAG-mCherry,-EGFP)Dym (termed RC-FrePe; stock \#029486, The Jackson Laboratory; Dymecki et al., 2010; Brust et al., 2014; Fig. 1). Triple-transgenic r2Hoxa2-cre, Pet1-Flpe, RC-FrePe $e^{+/-}$mice were generated by crossing doubletransgenic Pet1-Flpe hemizygous, RC-FrePe $e^{-1-}$ homozygous males to hemizygous $r 2$ Hoxa2-cre females. We also used a similar intersectional line $G t(R O S A) 26 S_{0} r^{\text {tm1.3(CAG-tdTomato,-EGFP)Pjen }}$ (termed $R C$ FLTG; Plummer et al., 2015) and comparable breeding strategy to visualize r2-Pet 1 and other Pet 1 subtractive fibers (stock \#026932, The Jackson Laboratory). To fluorescently mark Piezo2-Pet1 neurons, we generated triple-transgenic mice bearing the drivers Piezo2-GFP-IRES-cre (Woo et al., 2014) and Pet1-Flpe, combined with the conditional allele Gt(ROSA)26Sor ${ }^{\text {tm } 65.1(C A G-t d T o m a t o) H z e}$ (termed RC-Ai65; stock \#021875, The Jackson Laboratory; Madisen et al., 2012, 2015). Triple-transgenic r2Hoxa2-cre, Pet1-Flpe, RC$A i 65^{+1-}$ mice were generated by crossing double-transgenic Pet1Flpe hemizygous, $R C-A i 65^{-l-}$ homozygous mice with heterozygous Piezo2-GFP-IRES-cre mice.

Tissue preparation for immunohistochemistry and fluorescence in situ hybridization

All experiments used brain sections from mice perfused transcardially with $0.1 \mathrm{M}$ PBS, followed by $4 \%$ paraformaldehyde (PFA) as previously published (Brust et al., 2014). Brains were fixed overnight in 4\% PFA/ PBS for $24 \mathrm{~h}$ at $4^{\circ} \mathrm{C}$. For bouton neurochemical phenotyping or RNAscope experiments, tissue was then cryoprotected in 30\% sucrose/ PBS for $48-72 \mathrm{~h}$ at $4^{\circ} \mathrm{C}$ and then embedded in tissue freezing medium (Triangle Biomedical Services) for storage at $-80^{\circ} \mathrm{C}$. Tissue was sectioned into free-floating [ $20 \mu \mathrm{m}$ for single-molecule fluorescence in situ hybridization (FISH) and $40 \mu \mathrm{m}$ for immunohistochemistry (IHC)] coronal sections in series of six or eight. For retrograde tracing, sections at 50 $\mu \mathrm{m}$ thickness were cut on a vibratome (model VT1000S, Leica) and stored in $28 \%$ sucrose $30 \%$ ethylene glycol cryoprotectant at $-30^{\circ} \mathrm{C}$.

\section{Immunohistochemistry}

We performed IHC using the following antibodies and dilutions: anticalbindin (mouse monoclonal; 1:2000; catalog \#C9848, Sigma-Aldrich), anti-calbindin D-28k (mouse monoclonal; 1:3000; catalog \#300, Swant), anti-DsRed (rabbit polyclonal; 1:1000; catalog \#632496, Takara), antiGABA (rabbit polyclonal; 1:250; catalog \#ABN131, Millipore), anti-GFP (chicken polyclonal; 1:1500; catalog \#GFP-1020, Aves Labs), anti-Mouse IgG biotinylated (donkey polyclonal; 1:500; catalog \#A16021, Thermo Fisher Scientific), anti-neurokinin B (NKB; rabbit polyclonal; 1:500; catalog \#NB300-201SS, Novus Biologicals), anti-red fluorescent protein (rat monoclonal; 1:1000; catalog \#5f8, Chromotek), anti-Rabbit IgG biotinylated (donkey polyclonal; 1:200; catalog \#711-065-152, Jackson ImmunoResearch), anti-Reelin (goat polyclonal; 1:500; catalog \#AF3820, Novus Biologicals), 
A

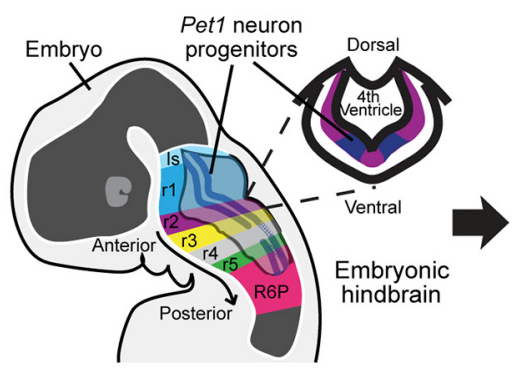

C

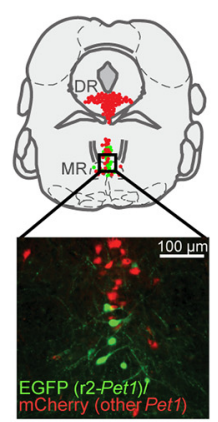

D
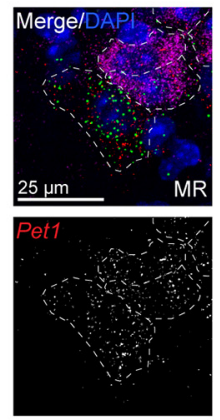

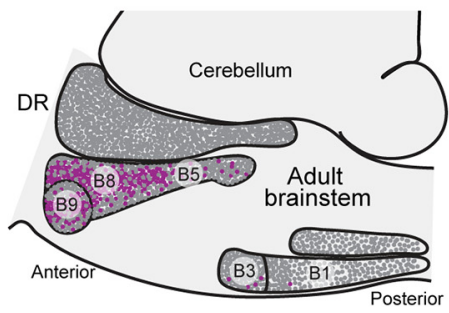

E

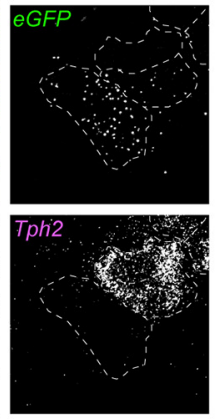

B

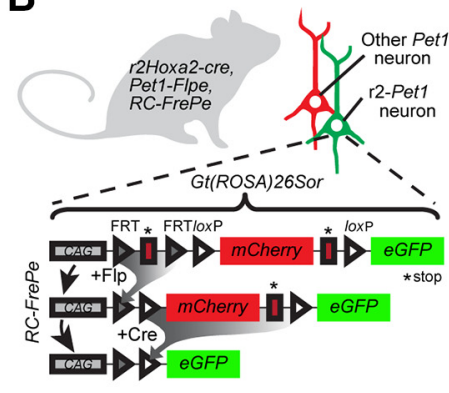

E'

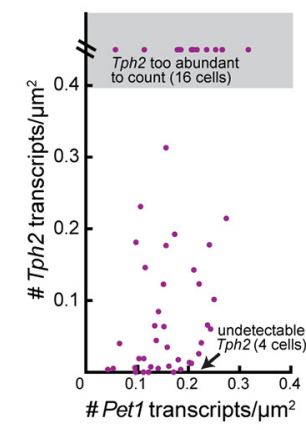

F

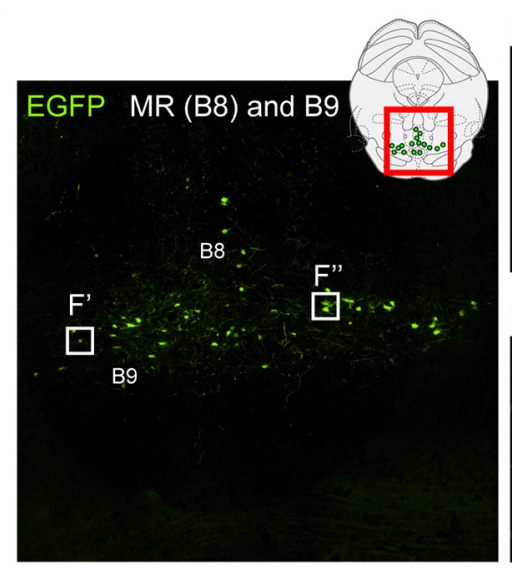

$F^{\prime}$ r2-Pet1 $1^{\text {Tph2-high }}$ cell
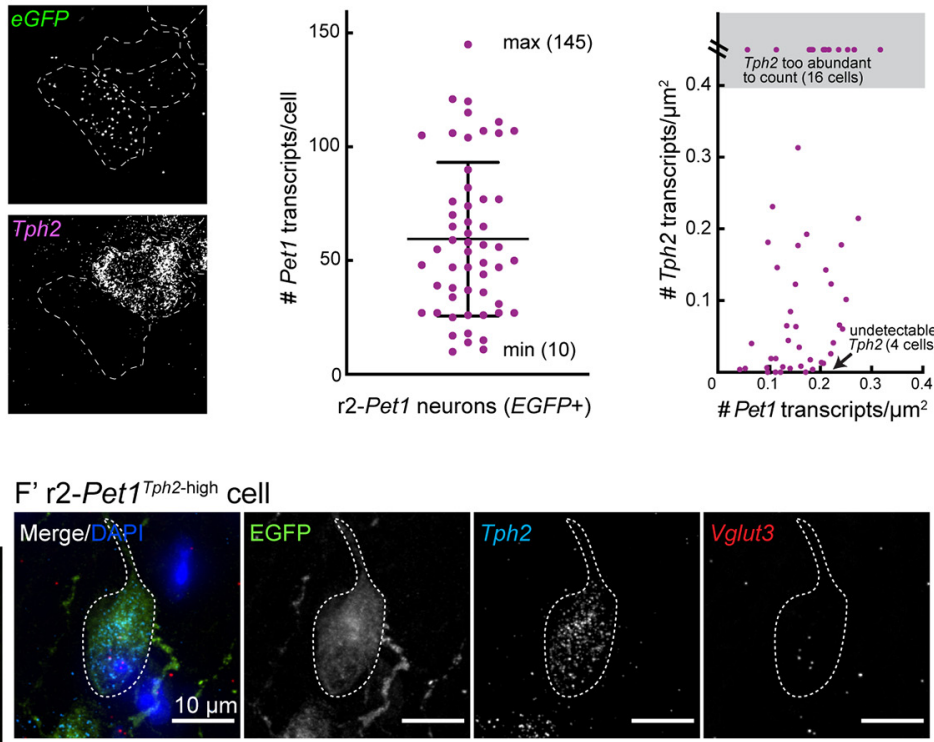

F" r2-Pet1 ${ }^{\text {vglut3-high }}$ cell
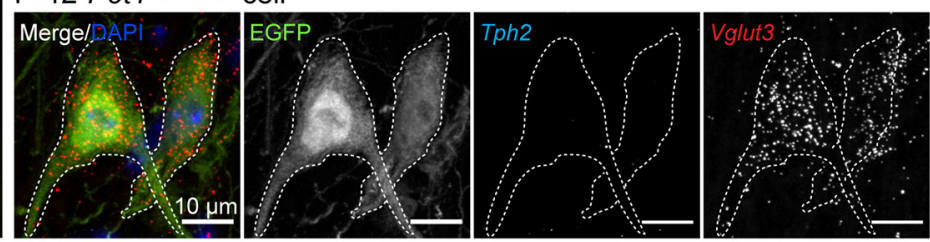

G

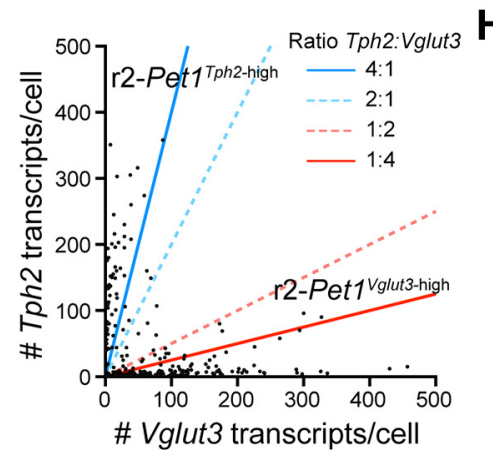

H

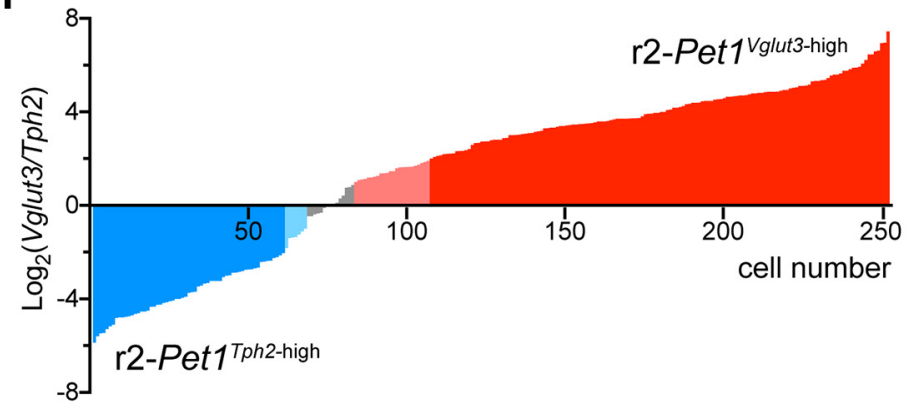

Figure 1. A, The developmentally specified r2-Pet1 neuron lineage comprises two transcriptomically polarized subtypes, one appearing more serotonergic (Tph2-high, Vglut3-low) and the other more glutamatergic (Tph2-low, Vglut3-high). r2-Pet1 cells are derived from progenitor cells in the 12 domain of the embryonic hindbrain that postmitotically express Pet1 and are intermingled with other lineages in the adult brainstem median raphe (B8 and B5) and B9 nuclei. B, C, We genetically access r2-Pet1 cells using ROSA26 (R26) knock-in dual Cre- and Flp-responsive intersectional alleles partnered with the r2- and serotonin lineage-specific drivers $r 2$ Hoxa2-cre and Pet1-Flpe, respectively. $\mathbf{D}-\boldsymbol{E}^{\prime}$, By fluorescence in situ hybridization performed on brain sections (D), all EGFP-labeled r2-Pet1 cells expressed detectable levels of Pet1 transcripts $(\boldsymbol{E})$, and Pet1 mRNA levels in r2-Pet1 cells weakly correlated with Tph2 transcript abundance $(r=0.3413$; $n=38$ cells, $p=0.036$, Spearman correlation; $\left.\boldsymbol{E}^{\prime}\right)$. $\boldsymbol{F}-\boldsymbol{H}, \mathrm{r2}-$ Pet1 cells comprise transcriptomically distinct subtypes, one appearing more serotonergic and expressing high levels of Tph2 and low levels of Vglut3 (r2-Pet $\left.7^{\text {Tph2-high }}\right)$, and one appearing more glutamatergic with the inverse pattern of gene expression ( $\mathrm{r} 2$-Pet $7^{\text {Vglut3-high }}$ ) as seen in RNA FISH-IHC fluorescent photomicrographs of tissue sections in which EGFP labeling marks 12 -Pet1 neurons $(\boldsymbol{F})$ and quantification of puncta counts per r2-Pet1 cell $(\boldsymbol{G}, \boldsymbol{H})$. Coronal brain schematics for all figures adapted from Franklin and Paxinos (2008) with permission (copyright Academic Press-Elsevier). 
anti-5-HT (goat polyclonal; 1:500; catalog \#ab66047, Abcam), anti-5-HT2C (goat polyclonal; 1:100; catalog \#sc-15081, Santa Cruz Biotechnology), anti5-HT3A (rabbit polyclonal; 1:500; catalog \#ASR-031, Alomone Labs), antisomatostatin (SST; rabbit polyclonal; 1:500; catalog \#HPA019472, SigmaAldrich), anti-TPH2 (rabbit polyclonal; 1:1000; catalog \#NB100-74555, Novus Biologicals), anti-VGLUT3 (rabbit polyclonal; 1:500; catalog \#135203, Synaptic Systems), anti-VGLUT3 (guinea pig polyclonal; 1:500; Synaptic Systems, catalog \#135-204), and anti-VIP (vasoactive intestinal peptide; rabbit polyclonal; 1:500; catalog \#20077, Immunostar).

Floating sections were incubated for $1 \mathrm{~h}$ at room temperature in a blocking solution of Tris-buffered saline (TBS) containing 3\% normal donkey serum (NDS; Jackson ImmunoResearch) and 0.3\% Triton X-100 (Sigma-Aldrich). Sections were then transferred to a solution containing primary antibodies diluted in TBS with $1 \%$ NDS and $0.3 \%$ Triton X-100 and incubated for $24 \mathrm{~h}$ at $4^{\circ} \mathrm{C}$ on a nutator mixer (BD Clay Adams). After three TBS washes, sections were transferred to a solution containing secondary antibodies diluted in TBS with $1 \%$ NDS and $0.3 \%$ Triton $\mathrm{X}-100$, and incubated in a light-protected chamber for $2 \mathrm{~h}$ at room temperature. Detection of 5-HT3A receptor followed previously established protocols (Chang et al., 2016), with ethanol dehydration before blocking. For detection of subtractive Pet1 fibers (non-r2-Pet1 fibers) in composite basket phenotyping experiments and for detection of calbindin-expressing interneurons in the basket target quantification experiments, we used a modified protocol. Briefly, amplification of signal was achieved by modifying the second day protocol using a biotinylated secondary antibody to primary antibody host IgG (see above) for $2 \mathrm{~h}$ at room temperature. Following manufacturer recommendations, the calbindin D$28 \mathrm{k}$ antibody was dissolved in TBS with $10 \%$ NDS. For DsRed, we dissolved the biotinylated antibody in TBS with $2 \%$ NDS and $0.1 \%$ Triton $\mathrm{X}-100$. To reduce nonspecific signal, we also used an avidin-biotin blocking kit (catalog \#SP-2001, Vector Laboratories) on IHC day 1 following manufacturer instructions. In general, for detection of primary antibodies, species-matched Alexa Fluor 488-, Alexa Fluor 568-, Cy5-, and Alexa Fluor 647-conjugated secondary antibodies (Jackson ImmunoResearch) were used. For visualizing biotinylated antibodies, we incubated sections with Alexa Fluor 555-conjugated streptavidin (1:500; catalog \#S32355, Thermo Fisher Scientific) during the same step as secondary antibodies. Sections were then washed three times in fresh TBS at room temperature as before, with $1 \mu \mathrm{g} / \mathrm{ml}$ DAPI (Life Technologies) added to the last wash step lasting $15 \mathrm{~min}$. Sections were mounted onto no. 1.5 coverslips (Electron Microscopy Sciences) and then attached to SuperFrost Plus Histologic Slides (Thermo Fisher Scientific) using Aqua-Poly/Mount medium (Polysciences) or ProLong Glass anti-fade mountant (Thermo Fisher Scientific).

Multiplexed fluorescent in situ hybridization

Single-molecule mRNA FISH (smFISH) was performed on fresh frozen or fixed tissue sections according to the manufacturer instructions (RNAscope, Advanced Cell Diagnostics; Wang et al., 2012). Sections were mounted onto SuperFrost Plus slides (Thermo Fisher Scientific) and dried on a slide warmer at $45^{\circ} \mathrm{C}$ for $30 \mathrm{~min}$ to promote section adhesion before proceeding with the RNAscope protocol recommended by suppliers (Advanced Cell Diagnostics). The RNAscope Manual Assay 2.5 was performed using the Multiplex Fluorescence Detection Kit (cata$\log \# 320851, \mathrm{ACD})$ and probes for eGFP (catalog \#400281, ACD), Fev (Pet1; catalog \#413241-C3, ACD), Tph2 (catalog \#Mm-318691-C2, CD), and Vglut3 (catalog \#Mm-431261-C1, ACD). For combined smFISH and IHC, following FISH, sections were then immunostained for EGFP as above and counterstained with DAPI. Slides were mounted using ProLong Gold Antifade Mounting media (Thermo Fisher Scientific) and no. 1.5 thickness glass coverslips.

Retrograde tracing experiments

To label r2-Pet1 cells that project to the hippocampus (HP), we injected three adult (two male, one female) r2Hoxa2-cre, Pet1-Flpe, RC-FrePe ${ }^{-/+}$ mice with AAV2-retro-CAG-tdTomato-WPRE virus (catalog \#59462AAVrg, Addgene) bilaterally into the dorsal hippocampus [coordinates: mediolateral (ML), $\pm 1.4 \mathrm{~mm}$; anteroposterior (AP), $-2.06 \mathrm{~mm}$; dorsoventral (DV), $-1.75 \mathrm{~mm}$ ]. The retrograde serotype of this virus allows infection of projection neurons that innervate the region targeted by viral injections and results in virus-induced marking of infected neurons by tdTomato. Three weeks postsurgery, mice were perfused and tissue processed as above. Sections were stained for EGFP and VGLUT3 or 5HT using a modified immunohistochemistry protocol. Briefly, sections were rinsed $3 \times$ in PBS, then $1 \times$ in PBS with $0.1 \%$ Triton X-100 (PBST; Sigma-Aldrich) before blocking in PBST with 5\% NDS for $2 \mathrm{~h}$ at room temperature. After incubating with primary antibodies for $48 \mathrm{~h}$ at $4^{\circ} \mathrm{C}$, sections are rinsed $3 \times$ in PBST and then incubated with secondary antibodies in PBST with 2\% NDS for $2 \mathrm{~h}$ at room temperature. After rinsing $3 \times$ in PBST (last rinse for $10 \mathrm{~min}$ with $1.5 \mu \mathrm{g} / \mathrm{ml}$ DAPI), sections were mounted on \#1.5 thickness coverslips and then adhered to slides with Prolong Glass mounting media.

Confocal and wide-field microscopy. Confocal imaging was performed using a Nikon Ti Inverted Spinning Disk Microscope equipped with a Yokogawa CSU-W1 Spinning disk scanhead with $50 \mu \mathrm{m}$ pinhole disk, a PI Z piezo stage insert, a TOPTICA iChrome MLE laser launch, and a Plan Apo $\lambda 60 \times 1.4$ numerical aperture (NA) oil objective for bouton neurochemical phenotyping and RNAscope experiments. For retrograde tracing experiments, a $20 \times$ Plan Apo $\lambda 20 \times / 0.75$ NA Air differential interference contrast (DIC) objective was used to generate tiled images. We also used this objective to quantify cell types targeted by r2Pet 1 baskets and to quantify the proportion of baskets that formed composites with other Pet1 fibers. Images were captured using an Andor Zyla 4.2 Plus sCMOS monochrome camera and Nikon Elements Acquisition Software AR 5.02. In all colocalization experiments, singlelabel controls were used to determine imaging settings to eliminate channel bleedthrough. Signal from the different channels was acquired sequentially using a Semrock Di01-T405/488/568/647 multi-bandpass dichroic mirror and bandpass emission filters for green (Chroma ET525/36m), red (Chroma ET 605/52m), and far red (Chroma ET 705/ $72 \mathrm{~m}$ ) channels. The $z$-stack acquisition was optimized to minimize axial shift between channels.

Several pericellular basket example images were taken using a laserscanning confocal Zeiss Axio Observer Z1 equipped with LSM780 Scan Head, motorized stage, and with either a Plan Apo $63 \times / 1.4$ Oil DIC III or Plan Apo $40 \times / 1.3$ Oil DIC III objective. These images were acquired using 405, 488, 561, and $633 \mathrm{~nm}$ laser lines, and a signal from each channel was acquired sequentially using Quasar PMT and GAaSP 32 Channel Spectral detectors. The range of wavelengths collected for each channel was determined using the Zeiss Zen Black (2011) software Smart Setup settings and compared with negative control slides. Voxel size was set to $0.21 \times 0.21 \times 1.13 \mu \mathrm{m}$.

Wide-field fluorescent micrographs were collected using a Zeiss Axioplan2 Upright Microscope with manual stage and an Axiocam 506 monochrome camera. Samples were illuminated with a Lumencor SOLA LED light engine. Signal from the different channels was acquired sequentially with FITC, DsRed, and Cy5 filter sets and used either a $10 \times / 0.45$ NA Air Plan Apochromat or $20 \times / 0.8$ Air Plan Apochromat objective. For all images, when brightness/contrast settings were adjusted using Image, identical settings were used for each channel applied uniformly across images within a given experiment. Images are presented as maximum intensity $z$-stack projections generated using Fiji (NIH; Schneider et al., 2012).

Bouton neurochemical immunophenotyping analysis and quantification. Histologic studies for bouton neurochemical phenotyping were performed using tissue collected from P50 to P100 male $(n=5)$ and female $(n=6)$ triple-transgenic $r 2 H o x a 2-c r e$, Pet1-Flpe, RC-FPSit mice from multiple litters. To determine whether r2-Pet1 neuron boutons contained VGLUT3, 5-HT, or both, tissue was stained for EGFP, VGLUT3, and 5-HT (see above for antibodies used). We collected four images of each ROI per animal when possible (mean number of images per region per animal $=3.82$ ) using a Nikon Ti spinning disk microscope with 1.4 NA $60 \times$ objective (see above). Colocalization of synGFP boutons with VGLUT3 and/or 5-HT was performed in a semiautomated manner in Imaris [Bitplane version 9.1.2 (January 29, 2018) Build 45902]. Briefly, each channel (synGFP, 5-HT, and VGLUT3) was converted into a spots object based on user-defined thresholds. If present, visual artifacts [e.g., highly autofluorescent cells of the olfactory bulb 
A
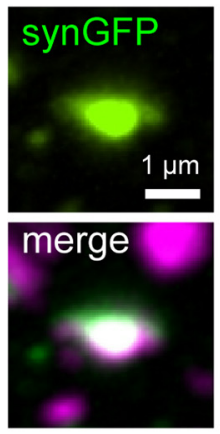

B

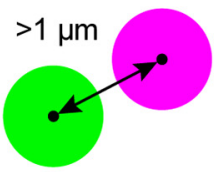

Not colocalized

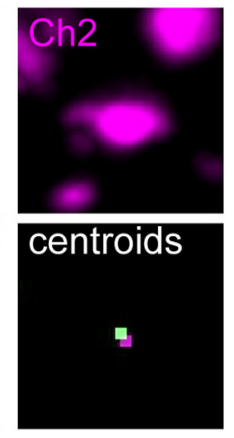

$<1 \mu \mathrm{m}$

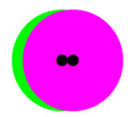

Colocalized
C Positive Control (double label)

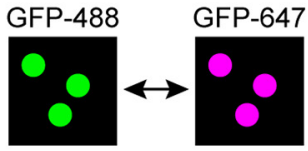

C"

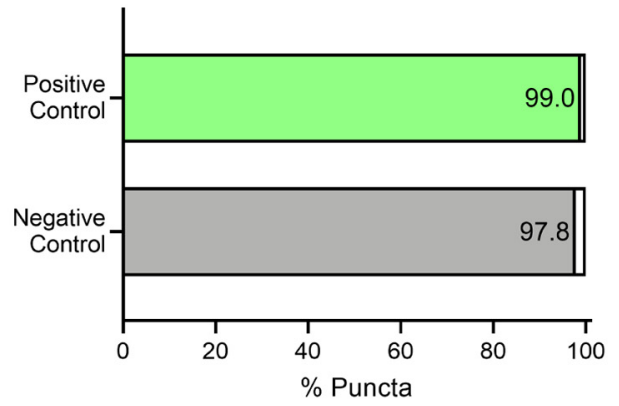

C' Negative Control (shuffle)

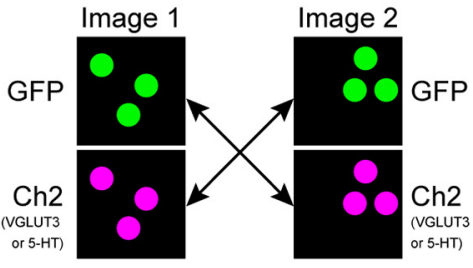

D
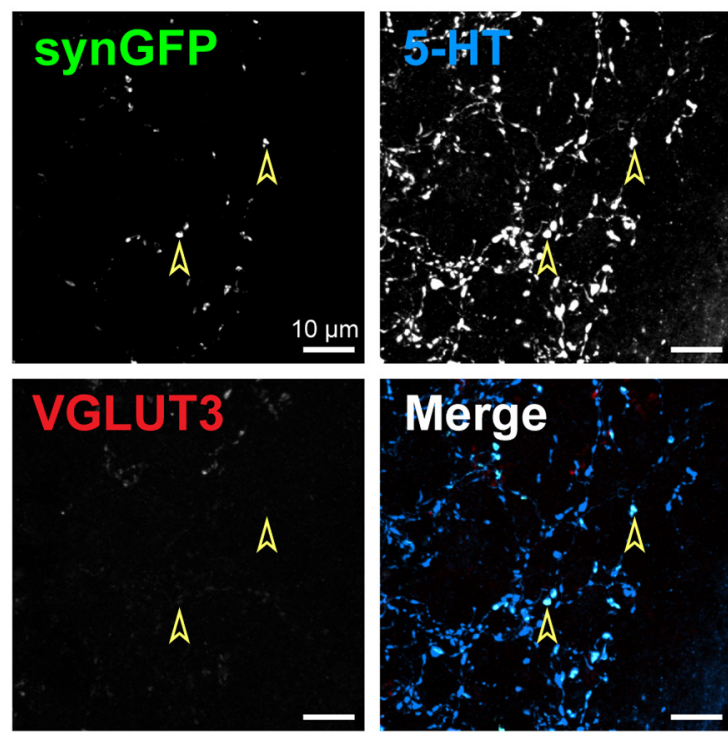

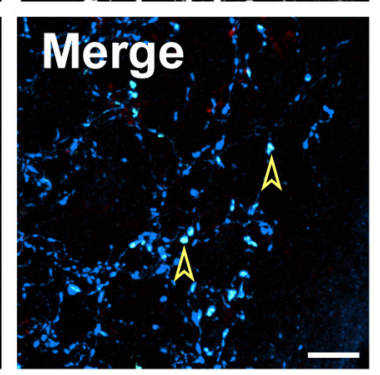

E

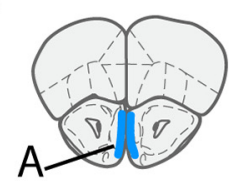

Olfactory Bulb Glomerular Layer Bregma $2.5 \mathrm{~mm}$

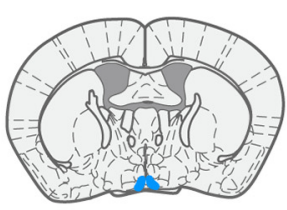

Suprachiasmatic Nucleus

Bregma $0.3 \mathrm{~mm}$

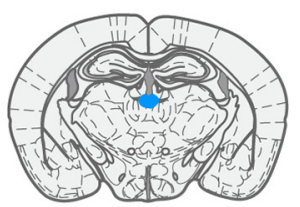

Paraventricular Nucleus of the Thalamus Bregma $-1.6 \mathrm{~mm}$

$\mathbf{F}$

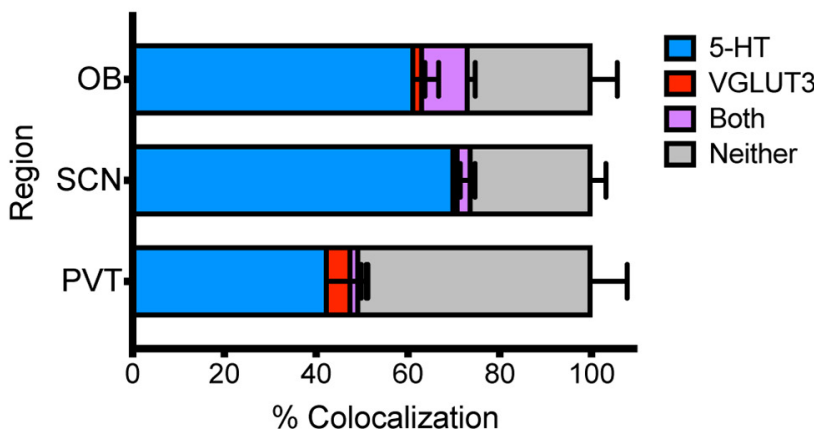

Figure 2. The $\mathrm{OB}, \mathrm{SCN}$, and PVT are innervated by $5-\mathrm{HT}^{+}$r2-Pet1 boutons. $\boldsymbol{A}, \boldsymbol{B}$, Example photomicrograph of a colocalized bouton $(\boldsymbol{A})$ and criteria for object-based colocalization $(\boldsymbol{B})$. $\boldsymbol{C}-\boldsymbol{C}^{\prime \prime}$, Positive control labeling of EGFP with multiple fluorophores $(\boldsymbol{C})$ and a negative shuffled channel control $\left(\boldsymbol{C}^{\prime}\right)$ were used to assess false-negative and false-positive rates, which are quantified in $\boldsymbol{C}^{\prime \prime}$. D, Fluorescent photomicrograph from the olfactory bulb glomerular layer showing r2-Pet1 boutons labeled by 5 -HT (arrowheads demarcate two of many) but not VGLUT3. $\boldsymbol{E}$, $\boldsymbol{F}$, Coronal schematics with regions demonstrating this innervation pattern in blue $(\boldsymbol{E})$ and quantification of colocalization $(\boldsymbol{F})$.

(OB)] were manually removed. The MATLAB (version 2017b) plugin "Colocalize Spots" was used to test for colocalization based on distance between synGFP centroids and 5-HT or VGLUT3 centroids. A distance threshold of $1 \mu \mathrm{m}$ was chosen empirically based on positive control (staining for synGFP with two secondary antibodies conjugated to different fluorophores) and negative control (randomly shuffling the synGFP channels of test images of a particular region to mismatch synGFP and VGLUT3 or synGFP and 5-HT signals) experiments. In these positive and negative control experiments, these analysis parameters resulted in a $1.03 \%$ false-negative rate and $2.16 \%$ false-positive rate (Fig. $2 A-C$ ). The same approach was used to neurochemically phenotype r2-Pet1 and other Pet1 boutons contributing to baskets in the lateral septum using images from adult r2Hoxa2-cre, Pet1-Flpe, RC-FrePe mice $(n=3)$.

Single-molecule mRNA FISH transcript quantification. For semiautomated analysis of mRNA transcript number in r2-Pet1 neurons, threecolor smFISH was performed to detect eGFP, Pet1, and Tph2 in triple- transgenic r2Hoxa2-cre, Pet1-Flpe, RC-FrePe mice from multiple litters. Image J was used to perform a 50 pixel rolling-ball background subtraction, to adjust window/level settings, and to generate threshold-transformed masks of each fluorescence channel. For each eGFP-marked r2Pet 1 cell, Tph 2 and Pet 1 transcripts were automatically quantified using the Analyze Particles function in Fiji (Schindelin et al., 2012) with a particle size of 1-10 pixels. Alternatively, a custom ImageJ analysis script (Okaty et al., 2020) was used to count Vglut3 and Tph2 mRNA puncta within $\mathrm{EGFP}^{+}$cell somata using the Default or MaxEntropy thresholds to segment cell somata and the Find Maxima function with a noise tolerance of 1000 to identify mRNA puncta. Analysis was performed on maximum intensity projections of $z$-stacks as this method reduced computational load and time, and produced results that were highly similar to a full 3D counting approach in Imaris (data not shown).

Quantification of cell somata in retrograde tracing experiments. For retrograde tracing experiments, images from virus-injected brains were 
analyzed by manual counting of $\mathrm{EGFP}^{+}$and tdTomato ${ }^{+}$cells using the Cell Counter plugin for Fiji (NIH). First, we selected display settings using single-label controls. We quantified the percentage of cells expressing VGLUT3 or 5 -HT for tdTomato ${ }^{+} / \mathrm{EGFP}^{+}$cells as well as for all tdTomato $^{+}$cells and all EGFP ${ }^{+}$cells.

Quantification of Pet1 composite baskets in septum. To quantify the proportion of septal cells targeted by r2-Pet1 baskets that also received boutons from non-r2 Pet1 cells, we analyzed triple-transgenic $n=4$ (2 male, 2 female) r2Hoxa2-cre, Pet1-Flpe, RC-FrePe mice and 1 r2Hoxa2-cre, Pet1-Flpe, RC-FLTG (male) mouse. First, we manually identified $\mathrm{EGFP}^{+} \mathrm{r} 2$-Pet1 baskets using only the green channel; then, each encased cell was manually reviewed for whether it was also targeted by $\mathrm{mCherry}^{+}$non-r2 Pet1 fibers. We quantified the proportion of $\mathrm{r} 2$ Pet1-targeted somata receiving (1) a non-r2 Pet1 basket (thus receiving a composite basket), (2) non-r2 Pet1 boutons that did not meet basket criteria, or (3) receiving no boutons from other Pet1 cells.

Quantification of cell somata targeted by r2-Pet1 baskets. To quantify the proportion of $\mathrm{VGLUT3}^{+}$and calbindin ${ }^{+}$somata encased by $\mathrm{r} 2$-Pet 1 baskets in the cortex, hippocampus, and septum, we executed the following. For VGLUT3 ${ }^{+}$somata and for calbindin ${ }^{+}$somata in hippocampus and septum, a user manually segmented cells in Fiji using the magic wand tool on a median-filtered image (radius: 5 for calbindin and 10 for VGLUT3). For calbindin ${ }^{+}$cells in the cortex, cell somata were amenable to automated segmentation using a custom Fiji macro. Briefly, the macro first corrected for uneven illumination by subtracting from the calbindin channel a Gaussian blurred image (radius, 100) of that channel. This was followed by a minor Gaussian blur (radius, 2) and autothresholding using the "Triangle" method. Resulting cell objects were filtered using the Analyze Particles function to limit cells to sizes between 40 and 300 $\mu \mathrm{m}^{2}$ area and 0.4-1.00 circularity; clumped cells were split using the Adjustable Watershed plugin with a tolerance of 1 . Finally, these images were manually reviewed, and any autofluorescent vasculature incorrectly counted by the macro as cells were removed. To examine cells in specific subregions of each image, another custom script sorted cells into userdefined regions of interest (ROIs). After segmentation, another custom script was used to quantify the area of r2-Pet1 boutons overlapping the quadrants of each cell body. Each cell was divided into quadrants with a gap at the center to prevent a bouton located at the center to be counted in all quadrants. The script then segmented $\mathrm{r} 2$-Pet1 fibers following the same automated method above (but without filtering for cell-sized objects) and quantified the bouton area in each quadrant. Cells were counted as receiving a basket if they had innervation in three or more quadrants of at least $1 \mu \mathrm{m}^{2}$ each and an overall innervation of $>12 \mu \mathrm{m}^{2}$. These parameters produced data that corresponded well to manually counting basket. All code is publicly available on Github in the repository "auto-basket-detector-2D" by user rsenft 1 .

\section{Statistical analyses}

Statistical tests were performed in Prism 8 (GraphPad Software). To explore the relationship between Tph2 and Pet1 expression, we performed a Spearman correlation on cells with countable Tph2 levels. To compare the neurochemical phenotypes of boutons in baskets against those outside baskets, we performed a within-animal two-tailed paired $t$ test. To compare the percentage of hippocampal-projecting r2-Pet 1 cells stained for 5-HT versus the percentage stained for VGLUT3, we performed a paired $t$ test. We also used paired $t$ tests to compare the neurochemical phenotypes of $\mathrm{r} 2$-Pet 1 versus other Pet 1 boutons contributing to composite baskets and to compare r2-Pet1 basket targets across regions. Results are reported as the mean \pm SEM, unless otherwise stated.

\section{Results}

\section{r2-Pet1 somata stratify by Tph2 versus Vglut 3 mRNA levels} even with abundant Pet1 transcripts

Previous molecular analyses of the r2-Pet1 neuron population suggested two categories of constituent cells based on neurotransmitter-related transcript levels: one cell subgroup was abundant in Tph2 mRNA; and the other was abundant in Vglut3
mRNA but demonstrating low or no detectable Tph2 transcripts (Okaty et al., 2015). This inverse relationship between Tph2 and Vglut3 mRNA abundance characterizing these two Pet1-expressing cell subgroups, referred to as $\mathrm{r} 2-\mathrm{Pet} 1^{\text {Tph2-high }}$ and $\mathrm{r} 2$ Pet $1^{\text {Vglut3-high }}$, contrasts with most other regions of the Pet $1^{+}$ brainstem raphe. More typical among raphe $P e t 1^{+}$cells are abundant levels of both Tph2 and Vglut3, or solely Tph2, with these serotonergic $\left(\mathrm{TPH}^{+}\right)$cells intermingled among various non-Pet1 raphe neurons, many of which are themselves glutamatergic. The distinctive, largely "one-or-the-other" neurochemical stratification distinguishing r2-Pet1 cell bodies suggests that the cognate axonal boutons might also show exclusivity for neurochemical phenotype (5-HT vs VGLUT3 indicative of glutamate release; Amilhon et al., 2010) and thus provide a means to resolve the efferent circuitry for each subgroup from within the broad innervation profile mapped for the r2-Pet1 population en masse (Bang et al., 2012). Before embarking on bouton phenotyping, however, we first set out to re-examine Tph2, Vglut3, and Pet1 transcript levels in r2-Pet1 neuron somata using the sensitive and specific approach of multiplexed FISH for mRNA detection.

We first performed single-molecule transcript analyses in tissue sections from triple-transgenic (r2Hoxa2-cre, Pet1-Flpe, RC$\mathrm{FrePe}$ ) mice in which EGFP protein and mRNA expression selectively marked the r2-Pet1 neuron population (Fig. $1 A-D$ ), on top of which Pet1 and Tph 2 mRNA could be visualized (Fig. 1D). As expected, given the use of the Pet1-Flpe driver, all eGFP transcript-marked cells showed Pet1 transcripts (fluorescent puncta), with a mean absolute count of $59.4 \pm 4.6$ Pet 1 mRNA puncta per cell, corresponding to an absolute range of 10-145 Pet1 transcripts/cell ( $n=54$ cells across three mice; Fig. $1 E$ ). This finding validates that r2-Pet1 neurons, indeed constituents of both subgroups, continue to express Pet1 in adulthood. Quantitative analyses of puncta representing individual Tph2 mRNA transcripts in these cells normalized to cell area showed that $\sim 30 \%$ of EGFP-marked r2-Pet1 neurons (16 of 54 cells) expressed high levels of Tph2 (Tph2 transcripts too numerous to count), while the remainder of cells (38) had few or no Tph2 puncta (Fig. $1 E^{\prime}$ ). For cells with countable levels of Tph2 puncta, area-normalized Tph 2 transcript levels exhibited a positive correlation with Pet 1 transcript counts (Spearman correlation, $r=0.3413 ; n=38$ cells, $p=0.036)$. Overall, these findings are consistent with our prior r2-Pet1 single-cell RNA sequencing (scRNAseq) results (Okaty et al., 2015).

Next, we explored the relationship between Tph2 and Vglut3 transcript abundance in the r2-Petl cells, using RNAscope FISH on tissue sections from a triple transgenic (r2Hoxa2-cre, Pet1Flpe, $R C-F r e P e$ ) mouse, but now visualizing the r2-Pet1 cells by immunofluorescent detection of EGFP protein (Fig. $\left.1 F, F^{\prime}\right)$. We counted Tph2 and Vglut3 puncta per $\mathrm{EGFP}^{+}$cell $(n=252$ r2Pet 1 cells) and calculated the base 2 logarithm of the Vglut $3 /$ $T p h 2$ transcript ratio. If $\geq 1$, we classified those cells as belonging to the $\mathrm{r} 2-\mathrm{Pet}^{\text {Vglut3-high }}$ group, and if -1 or less, to the $\mathrm{r} 2$ Pet1 $1^{\text {Tph2-high }}$ group (Fig. 1G,H), meaning that a ratio of at least 2:1 in transcript count is necessary to be classified as either cell type. By these criteria, we found $172(68.3 \%)$ cells as r2Pet1 ${ }^{\text {Vglut3-high }}$, and $65(25.8 \%)$ cells as r2-Pet $1^{\text {Tph2-high }}$. We also examined transcript counts normalized to cell area for cells classified into these groups, finding that $\mathrm{r} 2-\operatorname{Pet} 1^{\text {Tph2-high }}$ cells exhibited high Tph2 expression (mean, 0.9438 transcripts $/ \mu^{2}$; $95 \%$ CI, 0.86-1.03) and low Vglut3 expression (mean, 0.1096 transcripts $/ \mu \mathrm{m}^{2}$; $95 \% \mathrm{CI}, 0.084-0.14$ ), while $\mathrm{r} 2-$ Pet $^{\text {Vglut3-high }}$ cells expressed high Vglut3 (mean, 0.6308 transcripts $/ \mu \mathrm{m}^{2}$; $95 \%$ CI, 
A
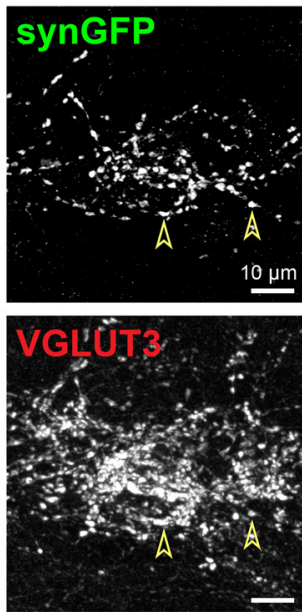

B
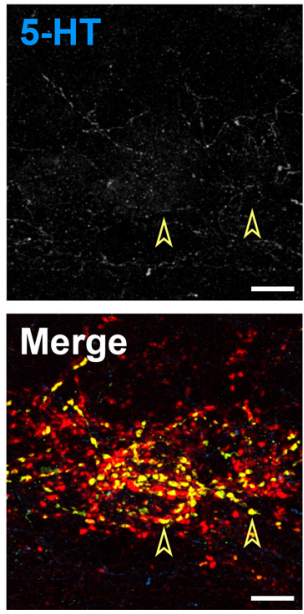

\section{C}

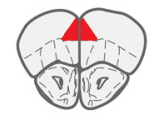

Prelimbic Cortex Bregma $2.5 \mathrm{~mm}$

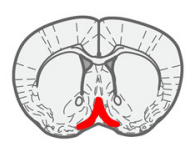

Diagonal Band Bregma $0.7 \mathrm{~mm}$

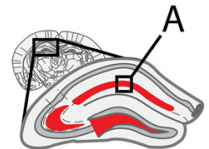

Rostral HP Bregma $-1.8 \mathrm{~mm}$

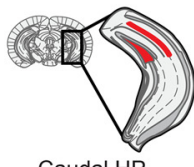

Caudal HP

Bregma $-3.1 \mathrm{~mm}$

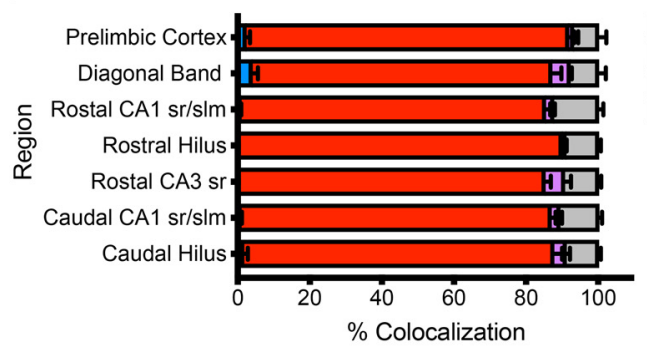

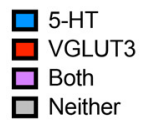

$\square$ VGLUT3

$\square$ Neither

Figure 3. The cortex, HP, and diagonal band are innervated by VGLUT3 ${ }^{+}$r2-Pet1 boutons. $\boldsymbol{A}$, Fluorescent photomicrograph from dorsal hippocampal CA1 sr/sIm showing r2-Pet1 boutons labeled by VGLUT3 but not 5-HT (arrowheads show examples). B, C, Coronal schematics with regions demonstrating this innervation pattern shown in red (B) and quantification of colocalization (C).

$0.58-0.69$ ) and low Tph2 (mean, 0.063 transcripts $/ \mu \mathrm{m}^{2}$; $95 \% \mathrm{CI}$, $0.051-0.075)$. A small subset of $r 2-$ Pet1 cells (5.9\%) contained an intermediate $V g l u t 3 / T p h 2$ transcript ratio or vice versa (i.e., a $<2: 1$ comparative enrichment of either Vglut 3 or Tph2. For simplicity, the present study approximates the molecular heterogeneity of $\mathrm{r} 2-$ Pet 1 neurons using the neuron subgroups descriptors of r2-Pet $1^{\text {Vglut3-high }}$ and $\mathrm{r} 2-P e t 1^{\text {Tph2-high }}$ cells, with the caveat that a small minority of $\mathrm{r} 2-\mathrm{Pet} 1$ neurons likely harbor an intermediate phenotype. Collectively, these RNAscope in situ hybridization findings are in line with the previously reported scRNAseq datasets (Okaty et al., 2015), confirming that the r2-Pet1 neuron group shows a segregation of somata into two subgroups based on expression of neurochemical pathway genes.

\section{Segregation anatomically of r2-Pet1 boutons by serotonin ${ }^{+}$ versus VGLUT3 ${ }^{+}$neurochemical identity}

To visualize and subsequently explore the anatomic distribution and neurochemical identity of r2-Pet1 neuron axonal boutons in the adult brain, we used the intersectional $R C$-FPSit allele (Niederkofler et al., 2016), which drives the expression of a synaptophysin-GFP (synGFP) fusion protein selectively in cells with a history of both Cre and Flp expression. Synaptophysin, a synaptic vesicle protein, enriches the fused GFP signal in both axon terminal and en passant boutons (De Paola et al., 2003; Kelsch et al., 2008; Li et al., 2010; Tripodi et al., 2011); both types of boutons have been shown to participate in vesicle-mediated neurotransmission (Beaudet and Descarries, 1981; Agnati et al., 1995; De-Miguel and Trueta, 2005; Trueta and De-Miguel, 2012). In triple-transgenic (r2Hoxa2-cre, Pet1-Flpe, RC-FPSit) mice, the synGFP robustly and selectively illuminated r2-Pet1 neuron axonal boutons compared with interbouton (i.e., intervaricose) axon segments. We observed the densest r2-Pet1 bouton distribution in the following brain areas: OB (glomerular layer), prelimbic cortex (superficial layers 1-3), suprachiasmatic nucleus of the hypothalamus (SCN), paraventricular nucleus of the thalamus (PVT), HP [present throughout many layers and highest at the stratum radiatum-stratum lacunosum moleculare ( $\mathrm{sr} / \mathrm{slm}$ ) border], medial and lateral septal nuclei (MS and LS, respectively), and diagonal band of Broca. This pattern of regions corroborates previous reports of fiber distribution from r2-Pet1 neurons (Bang et al., 2012).
Next, we probed synGFP-marked r2-Pet1 boutons for coimmunolocalization with 5-HT and VGLUT3. Brain sections (every eighth $40 \mu \mathrm{m}$ serial section from five male and six female P60 r2Hoxa2-cre, Pet1-Flpe, RC-FPSit mice) were immunostained and imaged on a confocal microscope, and the resulting images were analyzed using Imaris (Bitplane) to calculate the fraction of synGFP ${ }^{+}$boutons that were also immunopositive for 5-HT, VGLUT3, both, or neither, for four images per region per mouse (see Materials and Methods). We analyzed 66,435 synGFP ${ }^{+}$boutons across 13 forebrain subregions, for an average of 5,110 boutons per region and 465 boutons per region per mouse. Generally, we observed a segregation of bouton neurochemical identity by anatomic target site. In the glomerular layer of the olfactory bulb, the PVT, and the SCN, the r2-Pet1 boutons were largely immunopositive for 5-HT but not VGLUT3 (Fig. 2D-F). Furthermore, in the PVT, approximately half of the GFP-marked boutons lacked immunodetectable levels of even 5-HT. By contrast, dorsal cortical and hippocampal regions were largely innervated by VGLUT3-only r2-Pet 1 boutons $(86.3 \pm 0.94 \%$, across the seven regions; Fig. $3 A-C)$. Few $5-\mathrm{HT}^{+}$r2-Pet 1 boutons were observed ( $1.35 \pm 0.51 \%$ of all boutons across these regions), and a modest proportion lacked staining for either $(9.38 \pm 0.68 \%$ across these regions). These regions harbored synGFP ${ }^{-}, 5-\mathrm{HT}^{+}$ fibers, suggesting that these areas are also innervated by other (non-r2-derived) subtypes of serotonergic neurons, which is not surprising given that $\mathrm{r} 1$ - and $\mathrm{r} 3$-derived Pet1 serotonergic neuron cell bodies are also present in the MR (Jensen et al., 2008), and that dorsal raphe (DR) efferents also innervate these target regions (Azmitia and Segal, 1978). These results show that r2Pet 1 cells are in part responsible for the largely VGLUT3 ${ }^{+}$innervation of the hippocampus and cortex previously observed as arising from the median raphe (Jackson et al., 2008; Szónyi et al., 2016). In the dorsolateral septum (DLS) and medial septum (MS) and caudal stratum radiatum of the CA3 hippocampal subfield (cCA3-SR), the r2-Pet1 boutons were largely VGLUT3 only (66 $\pm 8.6 \%$ ), but $\sim 20 \%$ stained positive for both 5 -HT and VGLUT3 (20.4 \pm 5.2\%; Fig. $4 A-C)$. Furthermore, in the DLS, we also found 5 -HT-only r2-Pet1 boutons (8.04 $\pm 1.6 \%)$, making the DLS unique among examined regions in harboring all four types of r2-Pet1 boutons-5-HT-only, VGLUT3-only, co-positive, and negative 5-HT and VGLUT3. The DLS, and the septum 
A
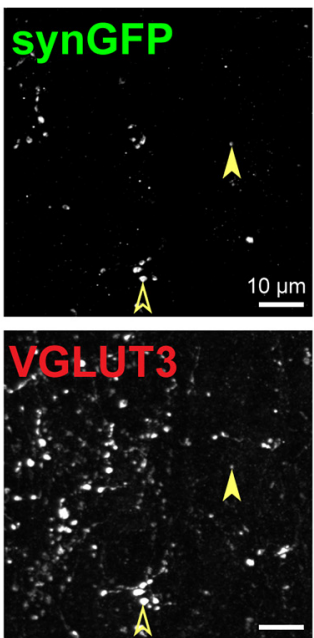
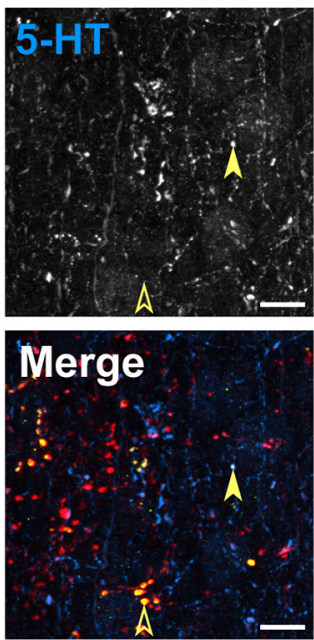

B
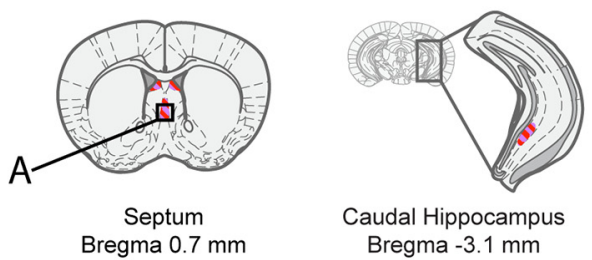

C

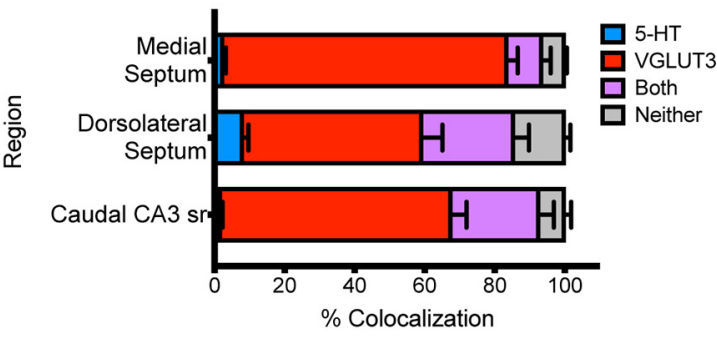

Figure 4. The medial and dorsolateral septum and caudal hippocampus are innervated by r2-Pet1 boutons that are either labeled by VGLUT3 alone or are 5-HT and VGLUT3 c0-positive. A, Fluorescent photomicrograph from the medial septum showing VGLUT3 ${ }^{+}$r2-Pet1 boutons (open arrowheads as examples) and 5 -HT ${ }^{+} / \mathrm{NGLUT3}^{+} \mathrm{r2}^{-P e t} 1$ boutons (filled arrowhead). $\boldsymbol{B}, \boldsymbol{C}$, Coronal schematics with regions demonstrating this innervation pattern in magenta $(\boldsymbol{B})$ and quantification of colocalization $(\boldsymbol{C}$.

more generally, also exhibited extensive non-r2-Pet1 5- $\mathrm{HT}^{+}$and VGLUT3 $^{+}$innervation, with many of these fibers immunopositive for both 5-HT and VGLUT3, suggesting another lineage of Pet 1 cells also innervates the septum and may be able to cotransmit 5-HT and glutamate.

\section{Driver Piezo2-cre coupled with Pet1-Flpe captured MR} boutons positive for serotonin but not VGLUT3

Given that the r2-Pet1 boutons found in the OB, SCN, and PVT were largely of the 5-HT-only class, we hypothesized that the source neuron subset was the r2-Pet $1^{\text {Tph2-high }}$ neuron subpopulation and not the r2-Pet $1^{\text {Vglut3-high }}$ subgroup. Alternatively, it is possible that a single r2-Pet1 neuron projects both serotonergic and glutamatergic boutons, for example, through differential axonal trafficking of mRNA and/or protein. To test our hypothesis, we devised means to intersectionally label the r2-Pet $1^{\text {TPh2-high }}$ neuron subpopulation. Single-cell transcriptomic analysis of Pet 1 MR neurons showed Piezo2 transcripts selectively enriched in r2-Pet1 ${ }^{\text {Tph2-high }}$ neurons compared with other Pet1 neurons (Okaty et al., 2015). This was further supported in the study by Ren et al. (2019) with the characterization of MR cluster 4, a Piezo $2^{+}$Tph2-high MR population expressing similarly modest levels of Vglut3 as the r2-Pet1 ${ }^{\text {Tph2-high }}$ group. Thus, we generated triple-transgenic Piezo2-GFP-IRES-Cre, Pet1-Flpe, RC-Ai65 mice to illuminate somata and axons of $\mathrm{r} 2-P e t 1^{\text {Tph2-high }}$ neurons selectively, which would also reveal the efferent target regions and allow neurochemical phenotyping for comparison with our generated bouton neurochemical maps for $\mathrm{r} 2$-Pet1 cells.

Cell bodies labeled in Piezo2-GFP-IRES-Cre, Pet1-Flpe, $R C$ Ai65 animals were restricted to the MR and were largely immunopositive for 5-HT (95.2 $\pm 4.76 \%)$, with a small fraction of cells copositive for VGLUT3 and 5-HT $(4.76 \pm 4.76 \%)$ and no cells immunopositive for VGLUT3 in the absence of 5-HT $(0.00 \pm$ $0 \%$; Fig. 5A). We observed fewer Piezo2-Pet1 neurons than would be expected of the entire $\mathrm{r} 2-P e t 1^{\text {Tph2-high }}$ population, and cell bodies were restricted to the midline, suggesting that they are a subset of the r2-Pet $1^{\text {Tph2-high }}$ group, or at least a subset accessible using this recombinase driver combination. We observed dense, $5-\mathrm{HT}^{+}$Piezo2-Pet1 fibers within the SCN $(73.2 \pm 3.27 \%$ $\left.5-\mathrm{HT}^{+}\right)$and the PVT $\left(60.5 \pm 3.16 \% 5-\mathrm{HT}^{+}\right.$; Fig. 5B,C). Piezo2-
Pet1 fibers were present, but less dense, in other regions where we observed 5- $\mathrm{HT}^{+}$r2-Pet1 innervation such as OB, LS, and MS (Fig. 5D-F). In these regions, the majority of Piezo2-Pet1 fibers were immunopositive for $5-\mathrm{HT}^{+}\left(\mathrm{OB}, 74.7 \pm 6.01 \% 5-\mathrm{HT}^{+}\right.$; LS, $56.2 \pm 4.97 \%$; MS, 71.6 $\pm 1.90 \%)$ but negative for VGLUT3 $\left(\mathrm{OB}, \quad 0.250 \pm 0.126 \% \mathrm{VGLUT}^{+}\right.$; $\mathrm{LS}, \quad 1.04 \pm 0.536 \%$; MS, $1.342 \pm 0.514 \%)$. Across all regions, a small percentage of Piezo2-Pet1 boutons were immunopositive for both VGLUT3 and 5 -HT, highest in the OB $\left(2.98 \pm 0.201 \% \mathrm{VGLUT3}^{+} / 5-\mathrm{HT}^{+}\right)$ and SCN $\left(2.87 \pm 0.437 \% \mathrm{VGLUT3}^{+} / 5-\mathrm{HT}^{+}\right)$. Upon exploring the target areas harboring VGLUT3-only boutons from r2-Pet1 neurons, we failed to detect innervation by the Piezo2-Pet1 neuron group (dorsal hippocampus shown; Fig. 5G). Together, these findings suggest that Piezo2-Pet1 neurons represent a r2Pet $1^{\text {Tph2-high }}$ population with projections largely restricted to the 5-HT-only SCN, PVT, and OB.

\section{Retrograde viral tracing from the dorsal hippocampus highlighted r2-Pet1 somata positive for VGLUT3 but not serotonin}

Given that the 2 -Pet 1 boutons found in the dorsal hippocampus were of the VGLUT3-only class, we hypothesized that the source neuron subset was the r2-Pet1 Vglut3-high neuron subpopulation and not the r2-Pet $1^{\text {Tph2-high }}$ subgroup. To test this, we injected bilaterally into the dorsal hippocampus (AP, -2.06 ; $\mathrm{ML}, \pm 2.06$; DV,$-1.75 \mathrm{~mm})$ of r2Hoxa2-cre, Pet1-Flpe, RC-FrePe mice $(n=3)$ a retrogradely transported, tdTomato-expressing adenoassociated virus (AAV) and analyzed the neurochemical phenotype (staining for either 5-HT or VGLUT3) of the tdTomato-labeled (and thus dorsal hippocampus-projecting) subset of r2Pet1 $\left(\mathrm{EGFP}^{+}\right.$) somata (Fig. 6). Worth noting, VGLUT3, in contrast to other VGLUTs, localizes both to axonal boutons and the somatodendritic compartment as well (Fremeau et al., 2004; Herzog et al., 2004), which we exploited here. To identify VGLUT3 $^{+}$cell bodies, we used a knock-out mouse-verified antibody (Schallier et al., 2011) that was previously used to label VGLUT3 $^{+}$somata in the raphe (Sos et al., 2017; Okaty et al., 2020) and found a distribution of VGLUT3-immunolabeled cell bodies consistent with prior anatomic studies describing the colocalization of Vglut $3 \mathrm{mRNA}$ and its protein in MR neurons 


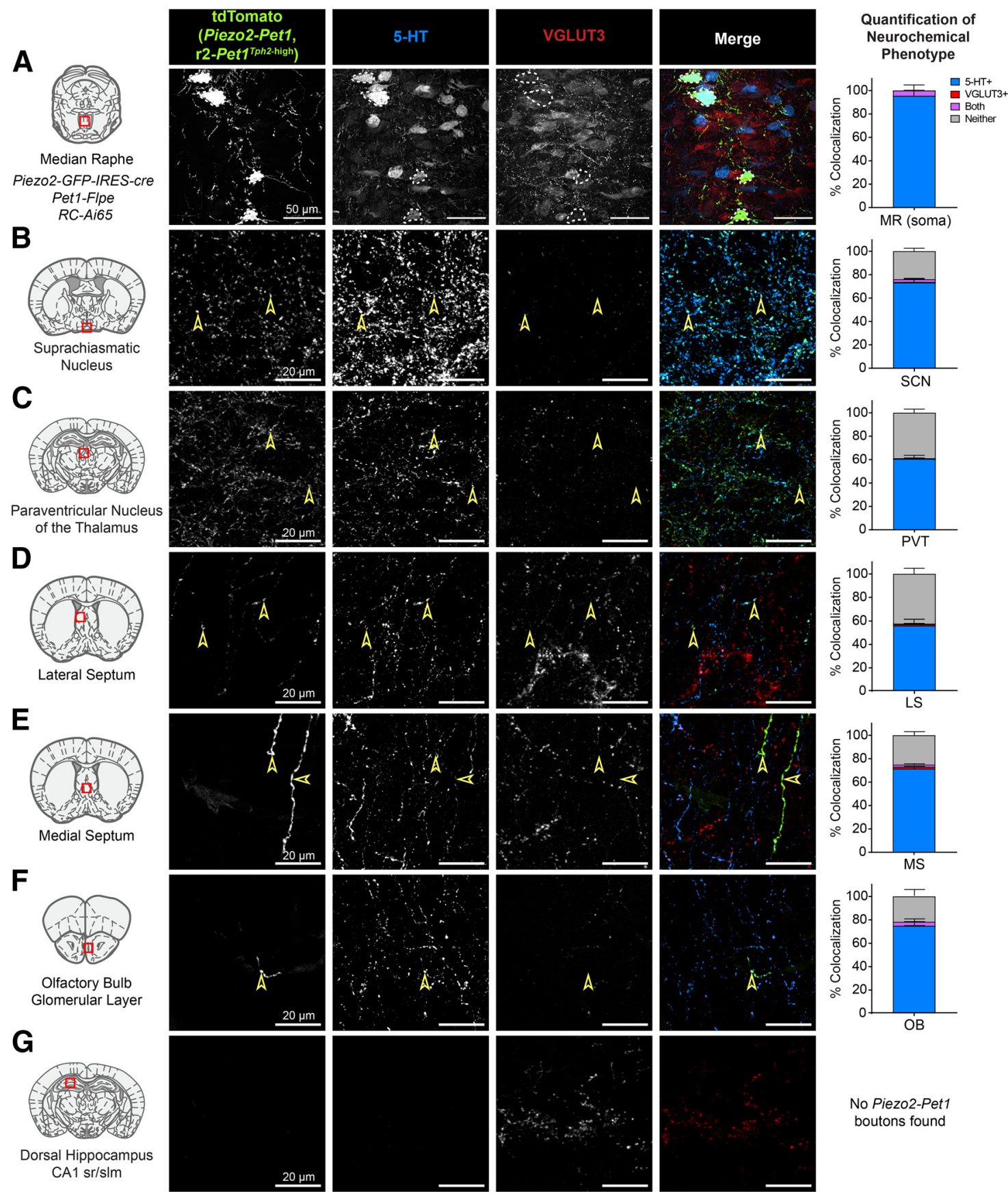

Figure 5. Piezo2-Pet1 neurons are of the $\mathrm{r2}$-Pet $\mathrm{T}^{\text {Th2-high }}$ subtype and harbor projections selectively to regions innervated by $5-\mathrm{HT}^{+}{ }_{\mathrm{r} 2-P e t 1}$ boutons. We previously identified Piezo2 as a marker of the r2-Pet1 ${ }^{\text {Thh2-high }}$ subtype (0katy et al., 2015). A, Piezo2-Pet1 cell bodies in the MR are labeled by 5-HT but not VGLUT3. B-F, Piez02-Pet1 neurons give rise to 5-HT ${ }^{+}$projections (arrowheads) in the SCN $(\boldsymbol{B})$, PVT $(\boldsymbol{C}$ ), lateral septum $(\boldsymbol{D})$, medial septum $(\boldsymbol{E})$, and olfactory bulb glomerular layer $(\boldsymbol{F})$. $\mathbf{G}$, By contrast, Piezo2-Pet1 cells do not innervate the dorsal hippocampus, a region shown to have predominately VGLUT3+ r2-Pet1 innervation.

(Herzog et al., 2004). We examined 1804 neurons, with 1263 $\mathrm{EGFP}^{+}$neurons, 629 tdTomato $^{+}$neurons, and 88 neurons colabeled for tdTomato and EGFP, with the latter indicating that our retrograde tracing conditions labeled a fraction of the r2-Pet1 cell group. Of the dual-labeled $\left(\mathrm{EGFP}^{+}\right.$, tdTomato $\left.^{+}\right)$r2-Pet1 somata, $82.3 \pm 1.46 \%$ stained immunopositive for VGLUT3, but only $2.0 \pm 2.0 \%$ stained positive for 5 -HT. Thus, the hippocampal-projecting $\mathrm{r} 2$-Pet1 somata were significantly more likely to be VGLUT3 ${ }^{+}$than $5-\mathrm{HT}^{+}(t=106.6, \mathrm{df}=2, p<0.0001$, paired $t$ test; Fig. 6E). This finding is in line with the r2-Pet1 bouton immunophenotype of VGLUT3-only mapped to the dorsal hippocampus. Of all retrogradely labeled tdTomato ${ }^{+}$cells in the

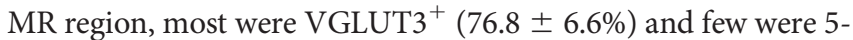
$\mathrm{HT}^{+}(9.6 \pm 3.86 \%)$, consistent with previous literature reporting a predominant VGLUT3 ${ }^{+}$innervation of the hippocampus from the MR (Jackson et al., 2008; Szónyi et al., 2016; Fig. 6E'). In contrast, when sampling all r2-Pet1 cells (EGFP labeled), a smaller fraction of cells was immunoreactive for VGLUT3 (52.0 \pm $2.19 \%$ ) and $28.74 \pm 0.94 \%$ were $5-\mathrm{HT}^{+}$(Fig. $6 E^{\prime \prime}$ ). These results demonstrate that the $\mathrm{r} 2$-Pet 1 cells that project to the dorsal hippocampus overwhelmingly express VGLUT3 in the soma, consistent with the neurochemical phenotype of the mapped r2-Pet1 boutons, suggesting that the source neurons are the r2Pet $1^{\text {Vglut3-high }}$. 
A

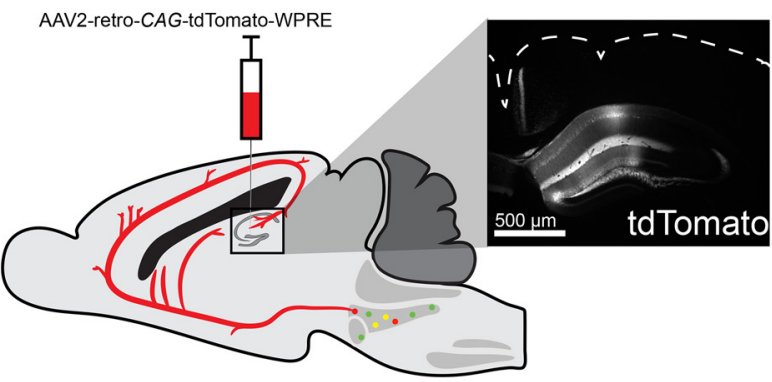

r2Hoxa2-cre Pet1-Flpe RC-FrePe

C

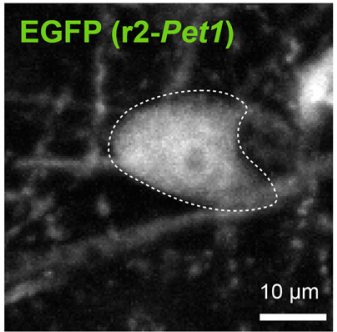

D

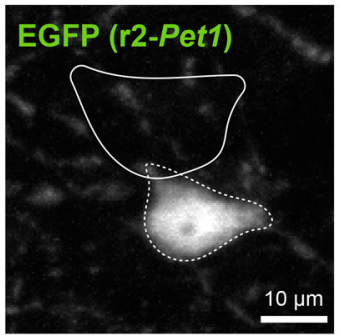

E

Retrogradely labeled r2-Pet1 neurons

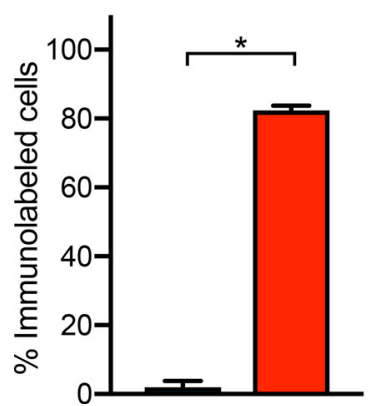

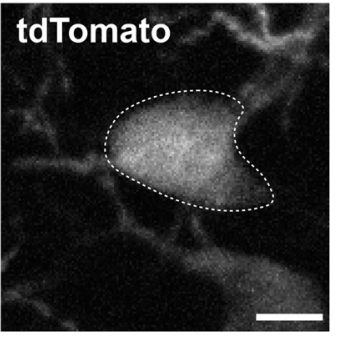

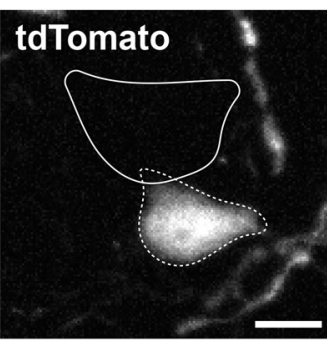

E'

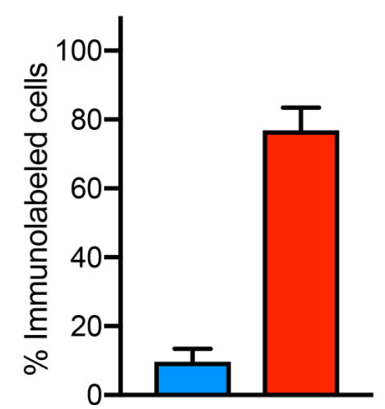

B

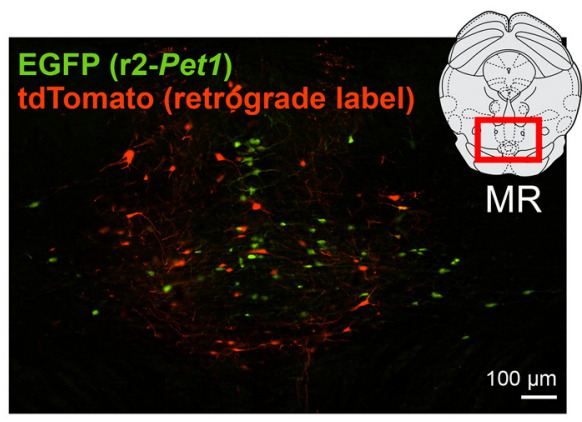

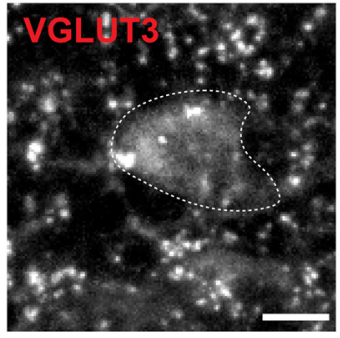
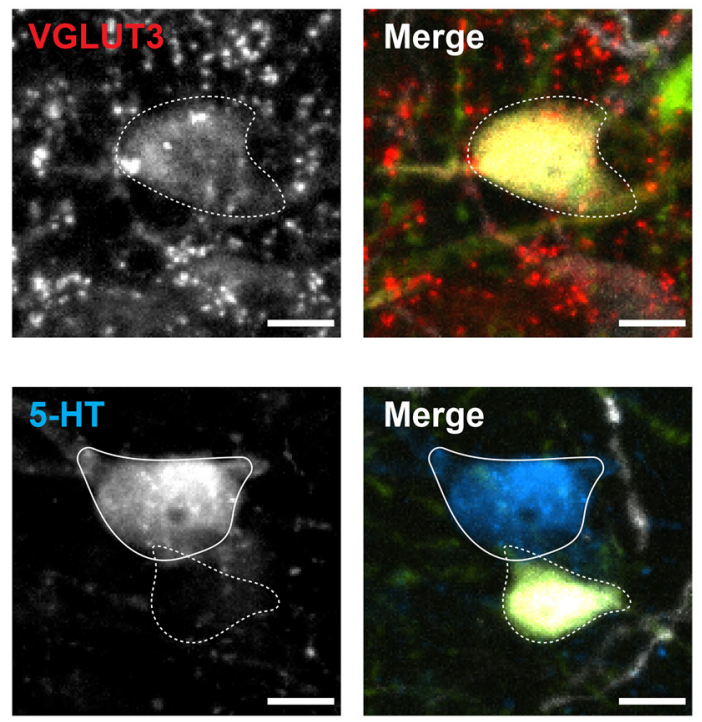

E"

All r2-Pet1 neurons

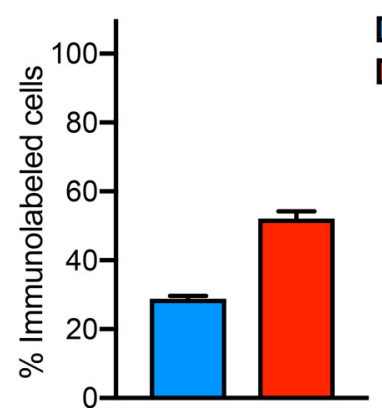

Figure 6. Hippocampal retrograde tracing shows that VGLUT3 ${ }^{+}$boutons in this region preferentially arise from $\mathrm{r2}$-Pet $7^{\mathrm{V} g \text { lut } 3 \text {-high }}$ neurons. $A$, Schematic showing injection, in the dorsal hippocampus (bilaterally) at the CA1 sr/slm border, of a retrograde serotype AAV expressing tdTomato under a CAG promoter performed in r2Hoxa2-cre, Pet7-Flpe, RC-FrePe mice expressing EGFP in r2-Pet1 cells. $\boldsymbol{B}$, Fluorescent photomicrograph showing the general localization of retrogradely labeled cells (tdTomato ${ }^{+}$) and r2-Pet1 cells (EGFP ${ }^{+}$) in the MR. C, D, r2-Pet1 cells that project to the hippocampus (EGFP ${ }^{+} /$tdTomato $^{+}$) have somata immunopositive for VGLUT3 (C) but not 5-HT (D). Hippocampus-projecting r2-Pet1 cells were more likely to be VGLUT3 ${ }^{+}$than 5 -HT ${ }^{+}$ $\left(\boldsymbol{E}, t=106.6, \mathrm{df}=2, p<0.0001\right.$, paired $t$-test). We also quantified VGLUT3 and 5-HT immunopositivity for all tdTomato ${ }^{+}$cells in the MR $\left(\boldsymbol{E}^{\prime}\right)$ or the entire population of r2-Pet1 cells $\left(\boldsymbol{E}^{\prime \prime}\right)$. Asterisks indicate statistically significant differences.

r2-Pet1 VGLUT3 ${ }^{+}$boutons comprise pericellular baskets in certain brain regions

In brain regions with $\mathrm{r} 2-\mathrm{Pet} 1 \mathrm{VGLUT3}^{+}$innervation, including the cortex (retrosplenial, cingulate, prelimbic, somatosensory, and motor cortex), hippocampus, and septal nuclei, we found r2Pet 1 boutons in dense arrays surrounding a target cell body and proximal dendrites, akin to previously described "pericellular baskets" (Fig. 7A-D; Köhler et al., 1982; Freund et al., 1990; Hornung et al., 1990; DeFelipe et al., 1991; Vu and Törk, 1992; Dinopoulos et al., 1993; Hioki et al., 2004; Riedel et al., 2008).
Operationally, we defined r2-Pet1 pericellular baskets when r2Pet 1 axons wrapped around more than half of the soma perimeter (or when no cell stain was present, the nucleus perimeter) or when boutons were closely apposing the soma on multiple sides such that dividing the cell into quadrants would result in boutons apposing the cell in all quadrants. Such pericellular basket structures rarely characterized $\mathrm{r} 2-\mathrm{Pet} 1$ boutons found in the $\mathrm{OB}$ and $\mathrm{PVT}$ and not at all in the SCN-all target regions harboring $\mathrm{r} 2$ Pet1 boutons of the 5-HT-only class. Additionally, Piezo2-Pet1 fibers representing a subset of the $\mathrm{r} 2$-Pet $1^{\text {Tph2-high }}$ subtype were 
A

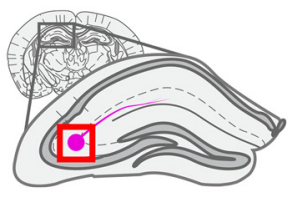

Hippocampus CA3 sr

B

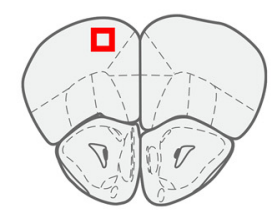

Motor Cortex

C

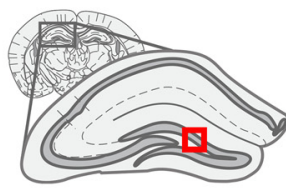

Hippocampus SGZ

D

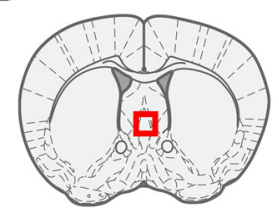

Medial Septum
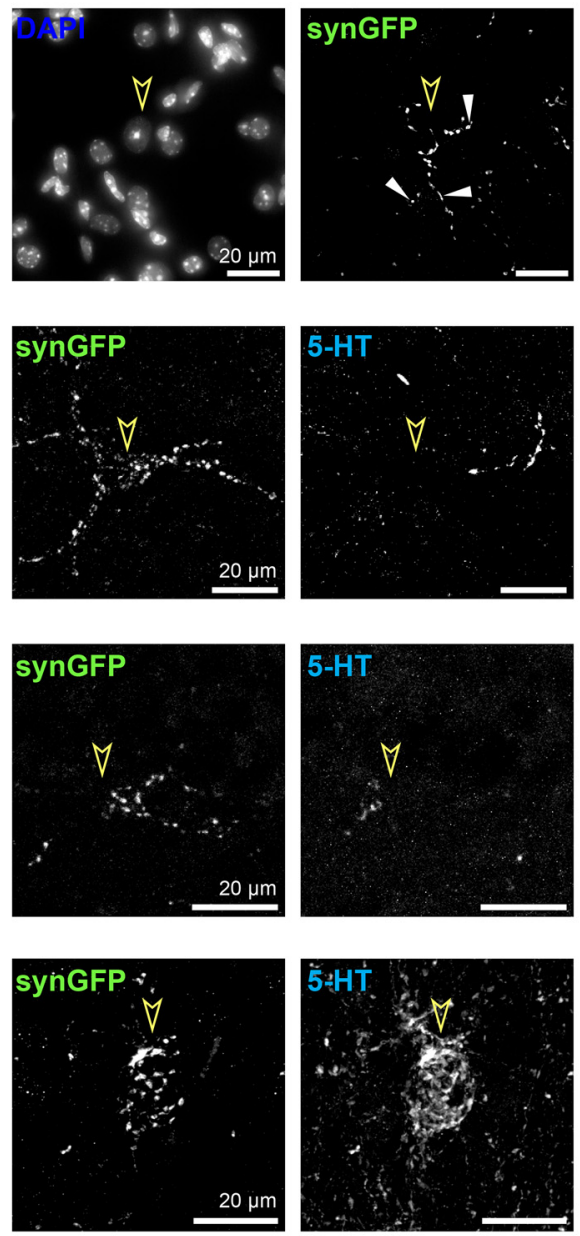
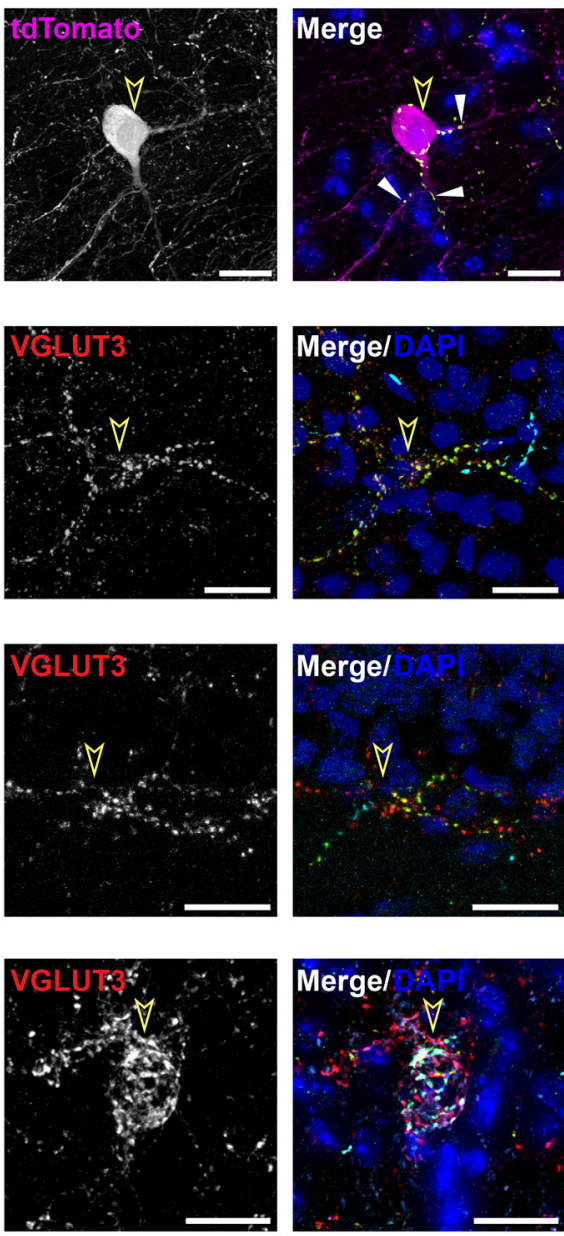

D'
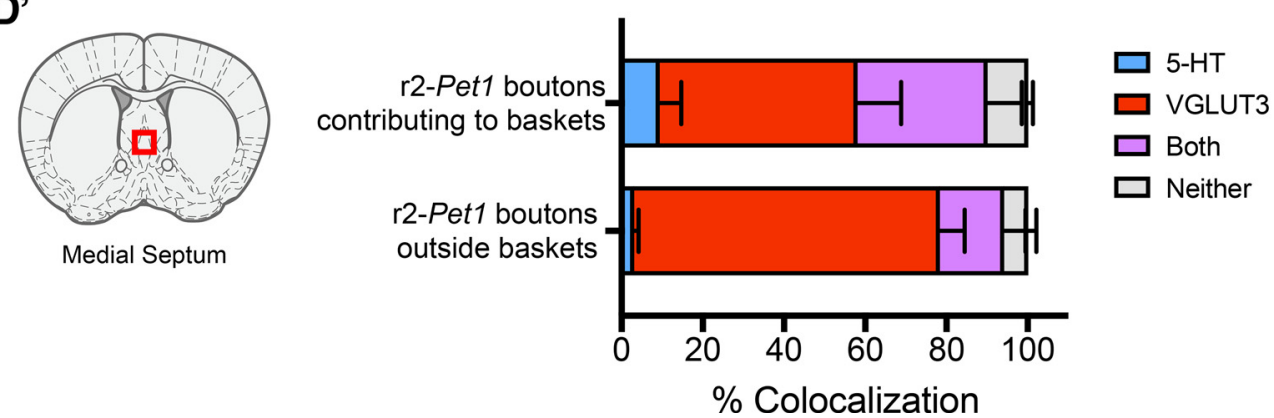

Figure 7. r2-Pet1 neuron boutons contribute to pericellular basket arrays in the cortex, hippocampus, and septum. $\boldsymbol{A}$, Fluorescent photomicrograph showing a basket comprising r2-Pet1 boutons surrounding the soma and proximal dendrites of a hippocampal CA3 cell retrogradely labeled by injection of AAV2-retro-CAG-tdTomato-WPRE into CA1. $\boldsymbol{B}, \boldsymbol{C}$, Fluorescent photomicrographs showing pericellular basket arrays in the motor cortex (B) and hippocampal subgranular zone (SGZ) (C) comprising VGLUT3 ${ }^{+}$r2-Pet1 boutons. $\boldsymbol{D}$, By contrast, $\mathrm{r}^{2}-$ Pet1 basket arrays in the medial septum comprise boutons that are $5-\mathrm{HT}^{-} / \mathrm{VGLUT3}^{+}$as well as copositive $5-\mathrm{HT}^{+} / \mathrm{NGLUT3}^{+} . \mathbf{D}^{\prime}$, In the medial septum, c0-positive 5 - $\mathrm{HT}^{+} / \mathrm{NGLUT3}^{+}$boutons are enriched in pericellular baskets, $(t=3.140, \mathrm{df}=6, p=0.020$, paired $t$ test). Operationally, we defined $\mathrm{r} 2$-Pet1 pericellular baskets as occurring when $r 2$-Pet1 axons surrounded $>50 \%$ of the perimeter of the cell body or nucleus and/or r2-Pet1 boutons closely apposed the target cell body in all four surrounding quadrants. Arrowheads are placed above the basket-targeted cell body.

not observed to make pericellular baskets. Thus, this specialized presynaptic basket structure may be feature specific to r2Pet $1^{\text {Vglut3-high }}$ cells, but not r2-Pet $1^{\text {Tph2-high }}$ cells.

We show in Figure 4 that r2-Pet 1 boutons in the septum are either $\mathrm{VGLUT3}^{+}$or $\mathrm{VGLUT3}^{+}$and $5-\mathrm{HT}^{+}$. We examined a subset of 11 medial septum images from r2Hoxa2-cre, Pet1-Flpe, $R C$-FPSit mice $(n=7)$, quantified in Figures $2-4$, and examined the neurochemical phenotypes of boutons belonging to baskets (manually defined as above) versus other boutons in the image innervating the same region but not contributing to baskets. Of the $\mathrm{r} 2$-Pet 1 boutons comprising pericellular baskets ( 473 boutons examined across all mice), $31.98 \%$ were co-positive, versus only $15.9 \%$ of boutons in nonbasket configurations (1300 nonbasket boutons examined across all mice; Fig. $7 D^{\prime}$ ), showing that $\mathrm{r} 2$ Pet1 boutons that contribute to baskets in the medial septum were significantly more likely to be co-positive for both VGLUT3 and 5-HT than nonbasket boutons in the same region ( $t=3.140, \mathrm{df}=6, p=0.020$, paired $t$ test). These results indicate that in the septum, boutons that contribute to baskets may be more likely to cotransmit 5-HT and glutamate. 
A

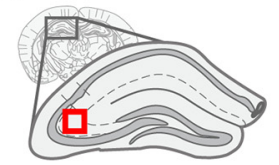

Hippocampus CA3 sr

B

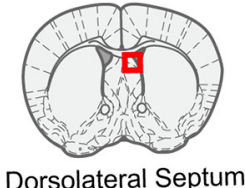

C

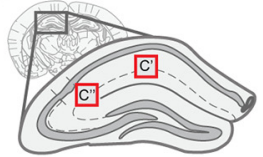

Rostral Hippocampus
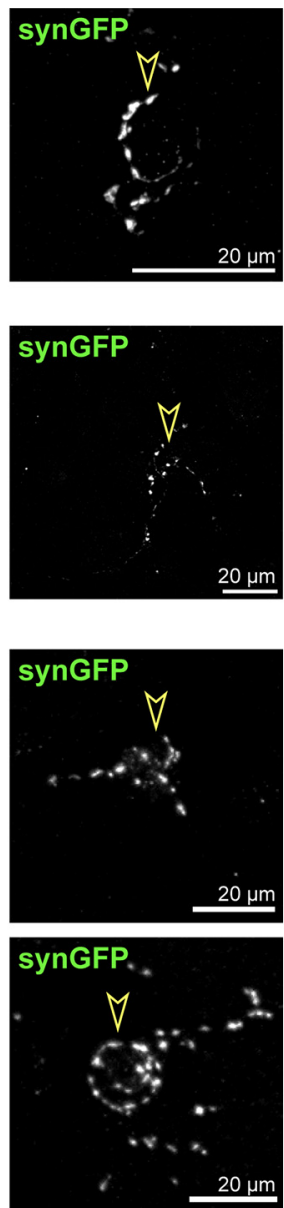

D

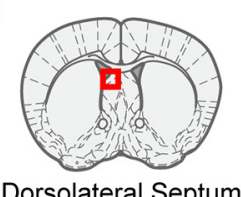

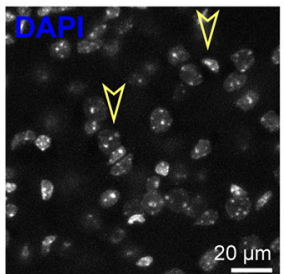
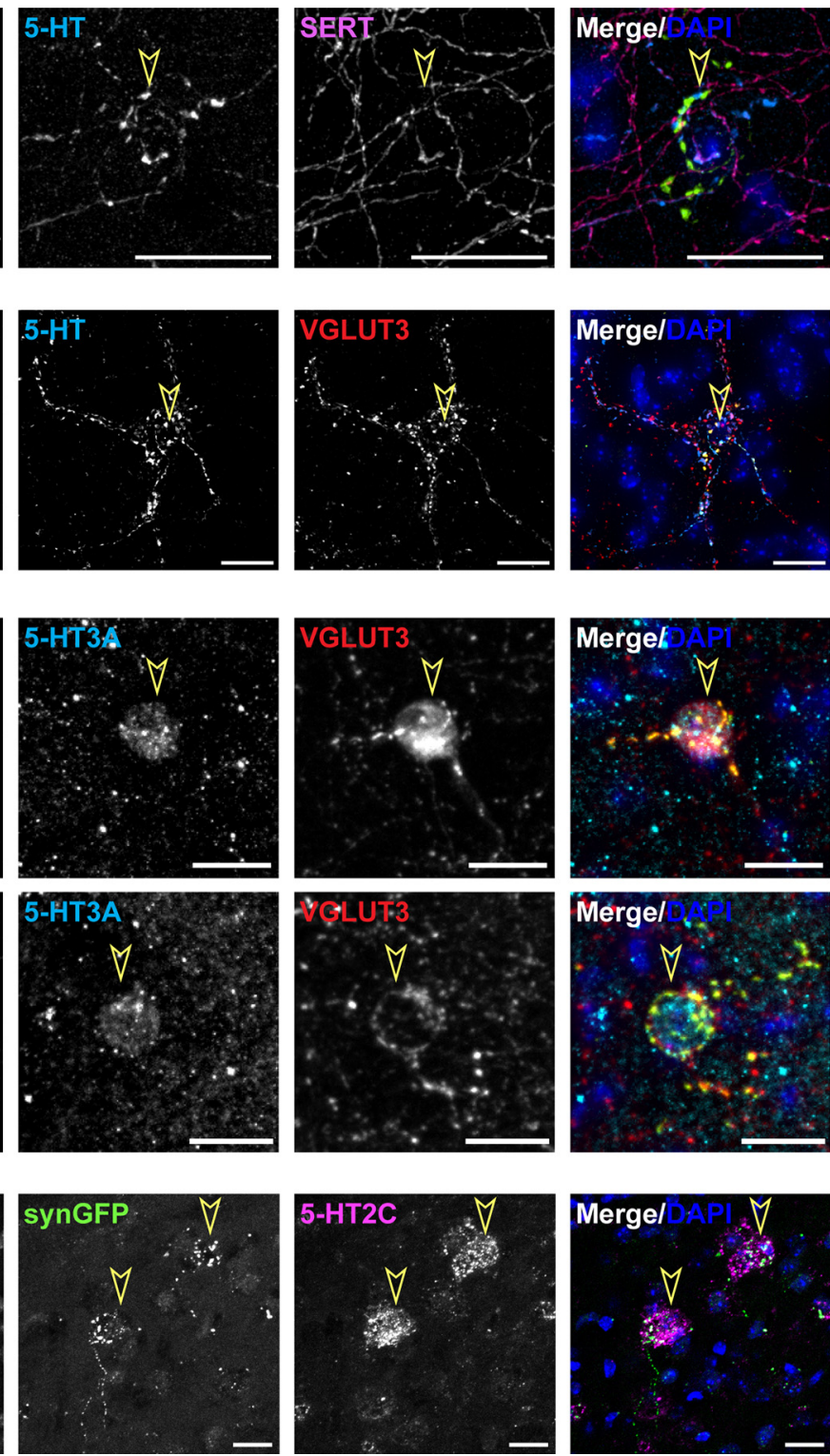

Figure 8. Cells targeted by r2-Pet1 pericellular baskets also receive 5-HT input. $\boldsymbol{A}, \boldsymbol{B}$, Fluorescent photomicrographs in hippocampus $(\boldsymbol{A})$ and dorsolateral septum $(\boldsymbol{B})$ showing baskets composed of both synGFP ${ }^{+}$r2-Pet1 boutons and non-synGFP-labeled 5-HT ${ }^{+}$boutons. $C$, $\boldsymbol{D}$, Consistent with the non-r2-Pet1 serotonergic innervation, some basket target cells expressed the 5 -HT receptors 5-HT3A $(\boldsymbol{C})$ and $5-\mathrm{HT} 2 \mathrm{C}(\boldsymbol{D})$. Arrowheads are placed above nuclei targeted by the $\mathrm{r2}$-Pet1 pericellular basket.

\section{Some pericellular baskets are comprised of boutons from multiple Pet1 neuron subtypes exhibiting distinct neurochemical profiles}

In some cases, our 5-HT and synGFP staining revealed synGFP ${ }^{-}$, $5-\mathrm{HT}^{+}$fibers converging on the same target cell as the synGFP ${ }^{+}$ r2-Pet1 boutons comprising a basket (Fig. 8A). This suggested that the former might arise from a non-r2-derived Pet 1 serotonergic neuron (i.e., a different subtype of serotonergic neuron). Such composite baskets (r2-Pet1 VGLUT3 ${ }^{+}$boutons and other 5 - $\mathrm{HT}^{+}$boutons) were found to be most prevalent in the stratum radiatum of the hippocampal CA3 subfield (Fig. 8A) and in the medial and lateral septal nuclei (Fig. $8 B$ ). In line with the observation that the non-r2-Pet1 fibers in these composite baskets were found to be $5-\mathrm{HT}^{+}$(or SERT ${ }^{+}$), we discovered that some of the postsynaptic target cells in the cortex and dorsal hippocampus expressed 5-HT3A (Fig. 8C), the ionotropic excitatory 5-HT receptor, and thus could be capable of receiving the basket-derived 5-HT signal. Moreover, the nature of the 5-HT3A receptor suggests that 5-HT may evoke fast excitatory synaptic firing (Lee et al., 2010) in these specific target cells. In the lateral septum, some basket-targeted cells were immunopositive for the slower signaling G-protein-coupled receptor 5-HT2C (Fig. 8D); none were immunopositive for 5HT3A (data not shown). Together, these findings suggest that 5-HT released from pericellular basket-associated boutons may act on distinct temporal scales across different brain regions.

To assess whether multiple Pet 1 neuron subtypes converge to form pericellular baskets around the same downstream cell, we labeled simultaneously r2-Pet1 fibers versus other (non-r2) Pet1 fibers with different fluorophores and examined basket composition in the septum, a region abundant in baskets. Triple-transgenic r2Hoxa2-cre, Pet1-Flpe, RC-FrePe ( $n=4,2$ males, 2 females) mice and one r2Hoxa2-cre, Pet1-Flpe, RC-FLTG ( $n=1$, male) mouse equally enabled this analysis, as each strain selectively marked $\mathrm{r} 2$-Pet1 somata and fibers intersectionally with 
A

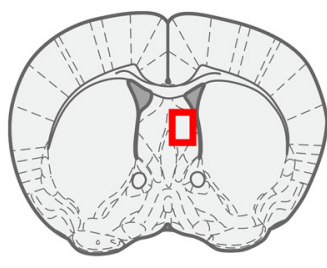

Intermediate Lateral Septum

A

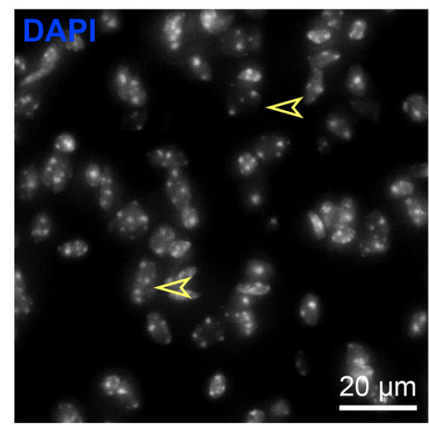

B

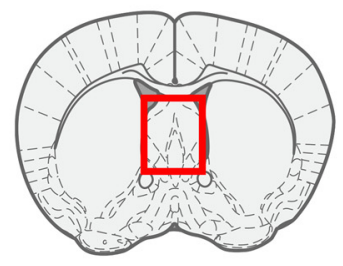

Septum
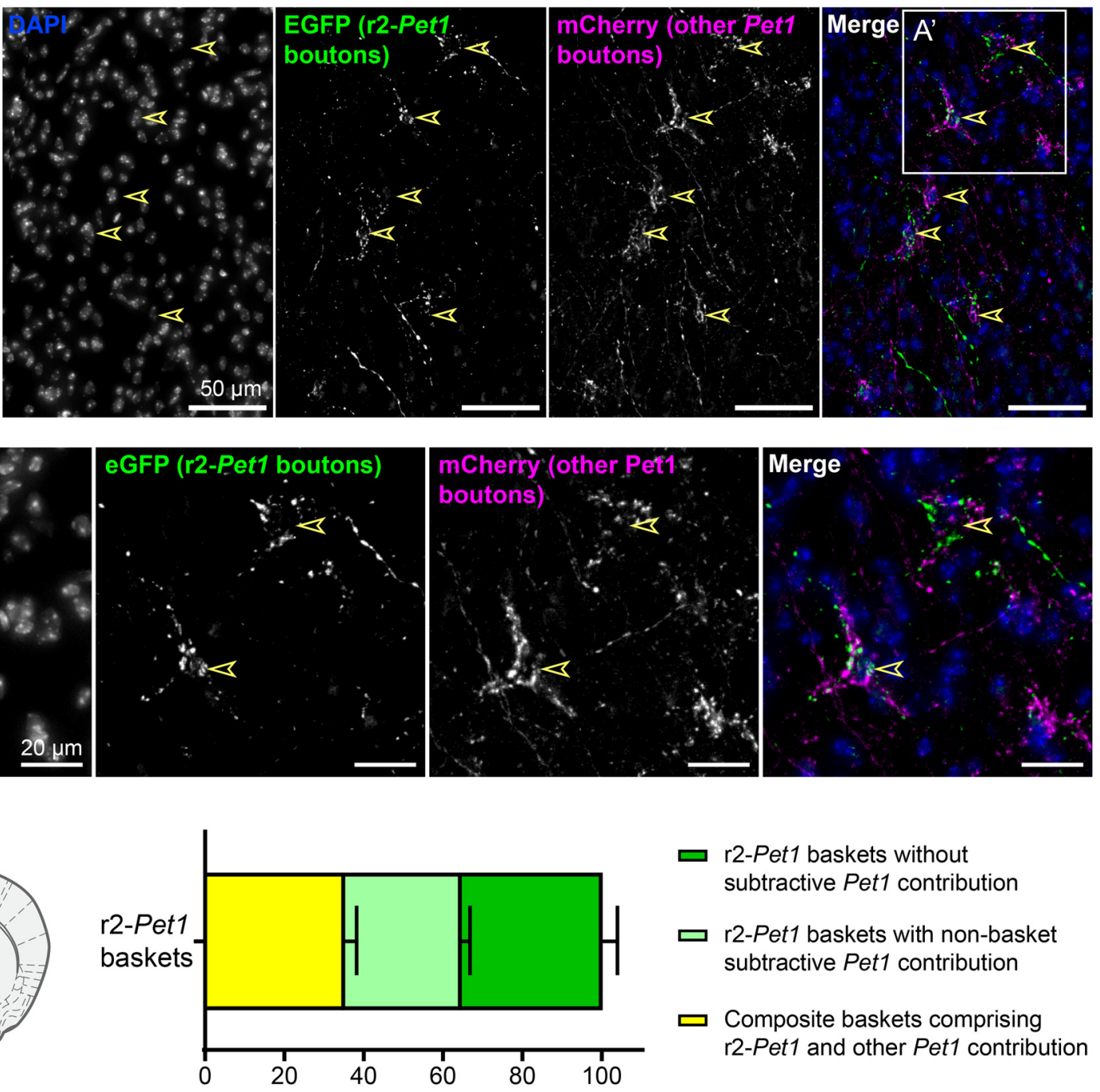

$\square$ r2-Pet1 baskets without subtractive Pet 1 contribution

$\square$ r2-Pet1 baskets with non-basket subtractive Pet1 contribution

$\square$ Composite baskets comprising r2-Pet1 and other Pet1 contribution

Figure 9. Multiple Pet1 neuron lineages contribute to composite pericellular baskets in the septum. $\boldsymbol{A}, \boldsymbol{A}^{\prime}$, Fluorescent photomicrographs showing composite baskets in the lateral septum in which r2-Pet1 axons (green) and other non-r2 Pet1 axons (magenta) converge onto the same targeted cell body, indicated by arrowheads. $\boldsymbol{B}$, Quantification of the proportion of $\mathbf{2}$-Pet 1 baskets that were composite with other Pet1 axons.

EGFP while simultaneously labeling other Pet1 cells with mCherry or tdTomato, respectively, as the subtractive population (Fig. 9A, $A^{\prime}$ ). We examined $163 \mathrm{r}$ 2-Pet1 pericellular baskets, finding many with nearby subtractive Pet 1 boutons closely apposing the same cell $(64.5 \pm 3.95 \%$ of r2-Pet1 baskets). Further, for $35.1 \pm 3.10 \%$ of $\mathrm{r} 2$-Pet 1 baskets, this subtractive Pet 1 contribution also formed a pericellular basket (Fig. 9B). For an additional third of r2-Pet1 baskets, there was no other Pet1 innervation closely apposing the targeted cell nucleus $(35.5 \pm 3.95 \%$ of baskets). These results demonstrate that $\mathrm{r} 2$-Pet 1 pericellular baskets are a heterogeneous group with a large portion converging with other Pet1 neurons to form composite pericellular baskets on the same downstream cell.

We next queried whether r2-Pet1 boutons in composite pericellular baskets differed neurochemically from other Pet 1 boutons contributing to the same baskets. We sampled 132 composite baskets (3,294 EGFP boutons and 2,672 mCherry boutons) of the lateral septum from adult r2Hoxa2-cre, Pet1-Flpe, $R C$-FrePe $(n=3,2$ male, 1 female) mice. In separate experiments, we immunodetected EGFP (r2-Pet1 boutons), mCherry (other
Pet1 boutons), and either 5-HT (Fig. 10A) or VGLUT3 (Fig. $10 B)$. Composite baskets showed no differences in amounts of r2-Pet1 EGFP boutons $(25.6 \pm 1.40)$ versus other Pet 1 mCherry boutons (19.8 \pm 1.52$)$. Baskets also contained additional boutons immunopositive for 5 -HT $(31.4 \pm 2.01)$ or VGLUT3 $(32.2 \pm$ 1.05) not captured using our transgenic fluorescent labeling approach. These boutons could be from Pet1 neurons missed by our drivers or immunohistochemical methods or alternatively, could reflect boutons from non-Pet1 neurons that form baskets around these same target cells (Fig. 10C,D). With respect to the Pet1-captured basket-forming fibers, we found the non-r2 Pet1 boutons were more likely to be $5-\mathrm{HT}^{+}(70.6 \pm 2.67 \%)$ than the r2-Pet 1 boutons $(45.9 \pm 3.42 \% ; t=12.7, \mathrm{df}=2, p=0.0062$, paired $t$ test; Fig. 10E). Both populations contributing to composite baskets were similarly likely to be VGLUT3 $^{+}$(r2-Pet1, $65.1 \pm 1.80 \%$; other Pet $1,58.6 \pm 4.61 \% ; t=1.02, \mathrm{df}=2, p=0.415$, paired $t$ test; Fig. 10F). Altogether, these findings demonstrate that (1) r2-Pet1 and other Pet1 neurons commonly form composite pericellular baskets around the same downstream target cells; and (2) in the lateral septum composite baskets are 
A

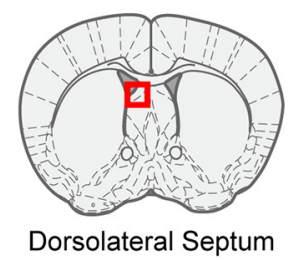

B

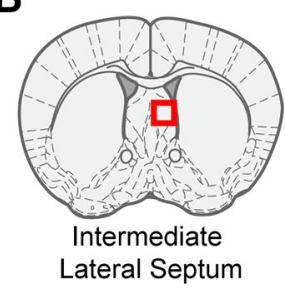

C

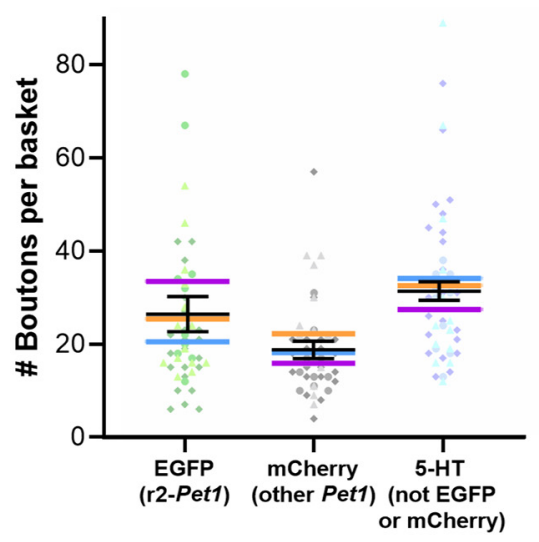

E

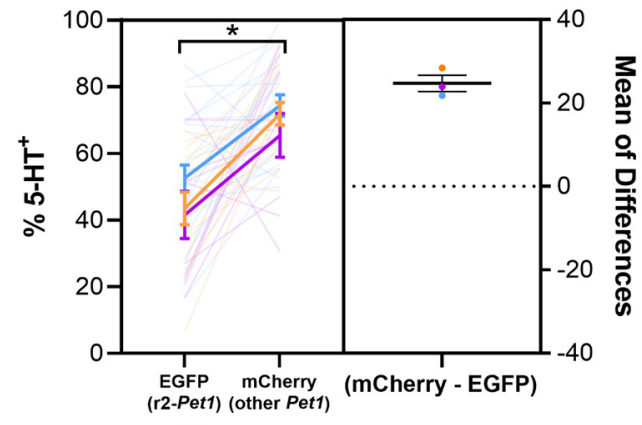

Bouton
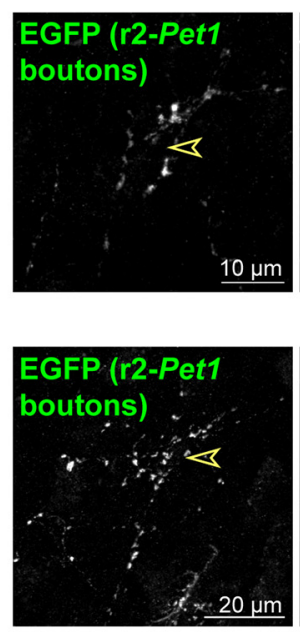
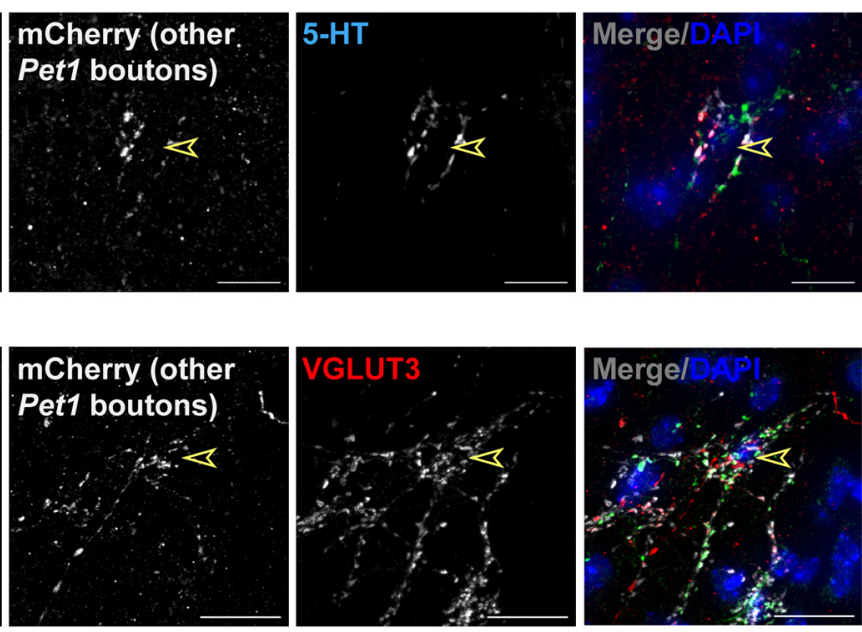

D

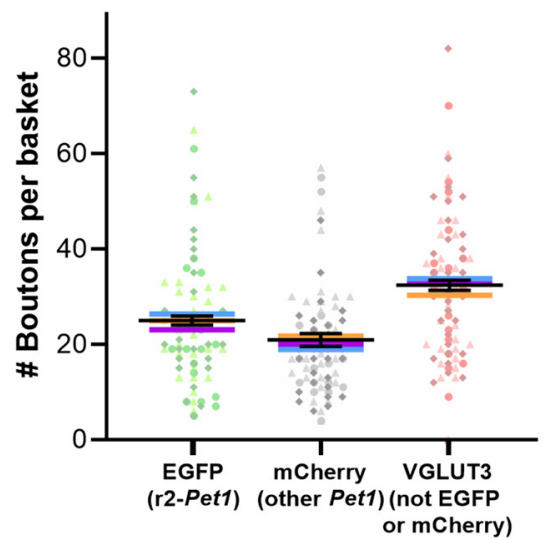

F

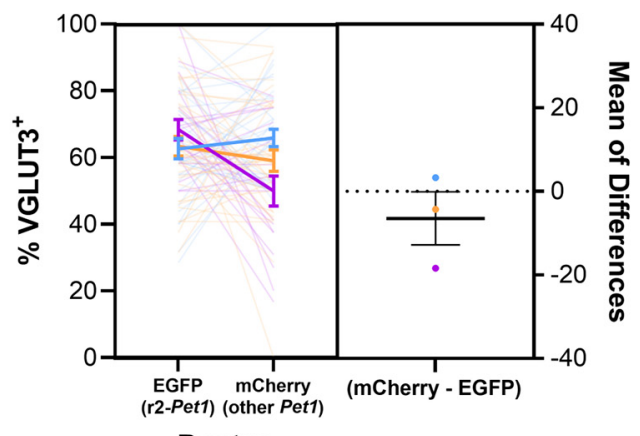

Bouton

Figure 10. In pericellular baskets, $\mathrm{r2}$-Pet1 boutons $\left(\mathrm{EGFP}^{+}\right)$and other Pet 1 boutons $\left(\mathrm{mCherry}{ }^{+}\right.$) are neurochemically distinct. $\boldsymbol{A}, \boldsymbol{B}$, Composite baskets with $\mathrm{r} 2$-Pet 1 boutons and other Pet 1 boutons in the lateral septum with colabeling for $5-H T(A)$ and VGLUT3 $(\boldsymbol{B})$. Arrowheads demarcate the location of the nucleus targeted by the pericellular basket. $\boldsymbol{C}, \boldsymbol{D}$, We found similar numbers of EGFP and mCherry boutons contributing to composite Pet1 lateral septum pericellular baskets. $\boldsymbol{E}, \boldsymbol{F}$, However, $\mathrm{r2}$-Pet1 boutons were less likely to be 5 - $\mathrm{HT}^{+}(t=12.7, \mathrm{df}=2$, $p=0.0062$, paired $t$ test; $\boldsymbol{E})$, but were similarly likely to be $\operatorname{VGLUT}^{+}(t=1.02, \mathrm{df}=2, p=0.415$, paired $t$ test; $\boldsymbol{F})$. The orange, purple, and blue lines indicate individual animal means, and individual measurements of baskets are indicated by differently shaped points in $\boldsymbol{C}$ and $\boldsymbol{D}$, and by lightly shaded orange, purple, and blue lines in $\boldsymbol{E}$ and $\boldsymbol{F}$. Asterisks indicate statistically significant differences.

neurochemically diverse, with $\mathrm{r} 2$-Pet 1 boutons less likely to be 5 $\mathrm{HT}^{+}$, but are similarly likely to be VGLUT3 ${ }^{+}$as other Pet1 fibers contributing to the same baskets.

\section{r2-Pet1 pericellular baskets target specific GABAergic neuron subtypes}

Next, we applied various cell subclass markers to further explore the identity of cells targeted by $\mathrm{r} 2$-Pet1 pericellular baskets. We found that $\mathrm{r} 2$-Pet1 pericellular baskets targeted $\mathrm{GABA}^{+}$neurons in both cortical regions and in the septum (Fig. 11A,B), a finding consistent with previous literature on the pericellular baskets formed by median raphe 5-HT neurons (Halasy et al., 1992; Hornung and Celio, 1992). Further, we found that target cells in the cortex and hippocampus compared with the septum could be differentiated by labeling with markers of specific interneuron classes. In the cortex and hippocampus, we observed r2-Pet 1 baskets around Reelin ${ }^{+}$and VIP ${ }^{+}$interneurons (Fig. 11C,D) as well as $\mathrm{VGLUT3}^{+}$interneurons that were sometimes positive for NKB (Fig. 11E). The latter group is likely to be the well described cholecystokinin $(\mathrm{CCK})^{+} / \mathrm{VGLUT3}^{+}$basket cell type, which in 
A

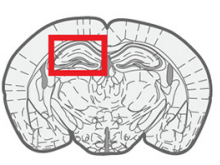

Dorsal Hippocampus

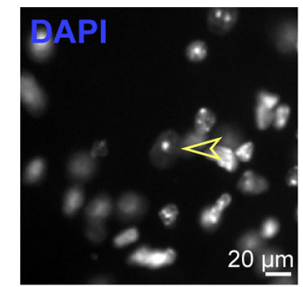

B

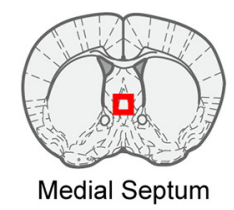

Medial Septum

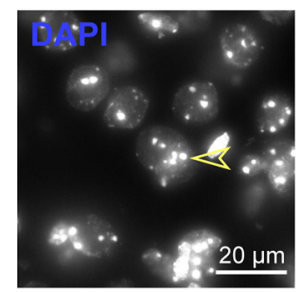

C

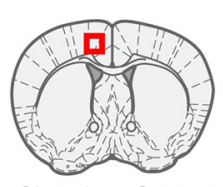

Cingulate Cortex
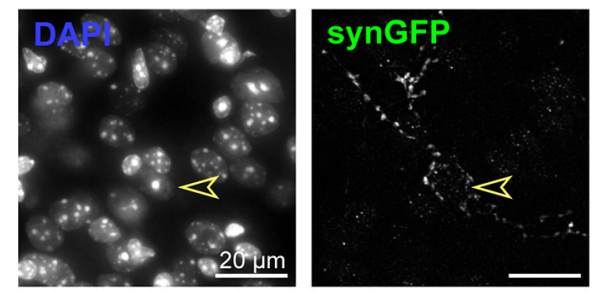
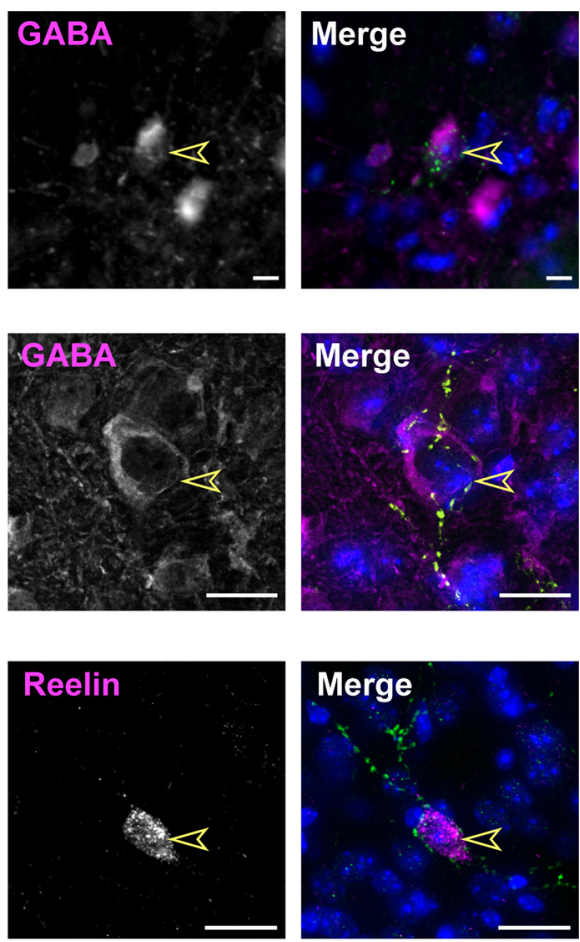

$D$

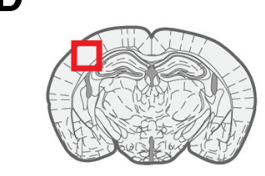

Somatosensory Cortex
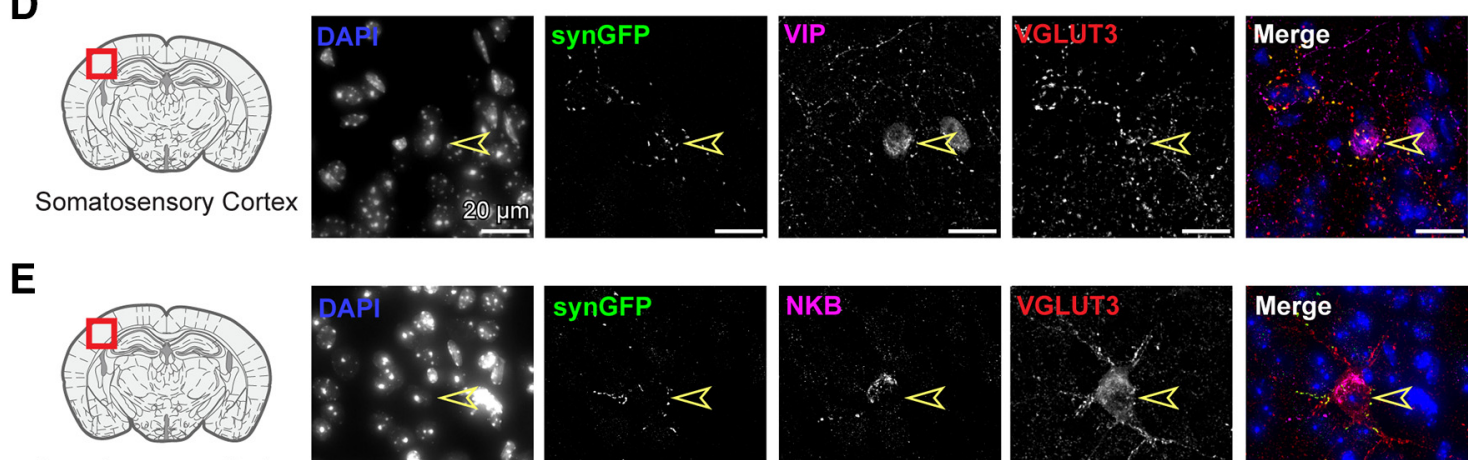

Somatosensory Cortex
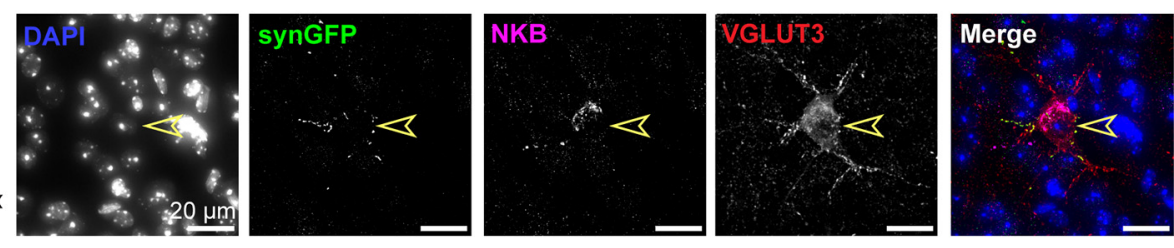

$\mathbf{F}$
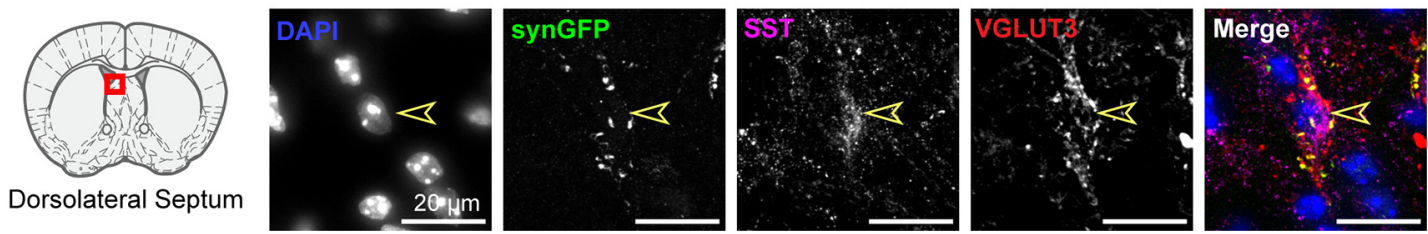

Figure 11. r2-Pet1 pericellular baskets target interneurons. $\boldsymbol{A}, \boldsymbol{B}$, Targets of pericellular baskets were overwhelmingly $\mathrm{GABA}^{+}$in dorsal hippocampus $(\boldsymbol{A})$ and medial septum $(\boldsymbol{B})$. $\boldsymbol{C}-\boldsymbol{F}$, Cortical and hippocampal basket targets were often Reelin ${ }^{+}\left(\boldsymbol{C}\right.$ ) calbindin ${ }^{+}, \mathrm{VIP}^{+}(\boldsymbol{D})$, or VGLUT3 ${ }^{+} / \mathrm{NKB}^{+}(\boldsymbol{E})$, whereas subcortical septal basket targets were sometimes SST ${ }^{+}(\boldsymbol{F})$. Arrowheads demarcate the cell body targeted by the r2-Pet1 pericellular basket.

turn forms pericellular baskets targeting cortical pyramidal neurons (Somogyi et al., 2004; Fasano et al., 2017; Booker and Vida, 2018; Harris et al., 2018). In contrast to the hippocampus and cortex, the targets of $\mathrm{r} 2$-Pet 1 pericellular baskets in the septum remain largely mysterious (Riedel et al., 2008), though some of these targeted cells express SST. In all regions, r2Pet1 fibers do not form baskets around parvalbumin ${ }^{+}$interneurons (data not shown), consistent with previous studies from the MR (Freund et al., 1990; Hornung and Celio, 1992; Papp et al., 1999) and suggesting the r2-Pet1 pericellular basket regulates specific subtypes of interneurons and possibly GABAergic projection neurons in the septum.
We then quantified the prevalence of $\mathrm{r} 2$-Pet 1 baskets on two interneuron classes in cortex, hippocampus, and septum: calbindin $^{+}$and $\mathrm{VGLUT3}^{+}$somata. We examined sections containing dorsal hippocampus and septum in adult r2Hoxa2-cre, Pet1-Flpe, RC-FPSit mice ( $n=6,3$ male and 3 female). Using a combination of manual and automatic segmentation combined with automatic detection of baskets (see Materials and Methods), we quantified the proportion of calbindin ${ }^{+}$and VGLUT3 ${ }^{+}$ somata receiving a $\mathrm{r} 2$-Pet 1 basket. We observed that VGLUT3 ${ }^{+}$ somata were most prevalent in layer 2 and layer 6 in cortex, and in the sr and sr/slm border region in hippocampus, which is in line with previous studies (Fremeau et al., 2004; Fig. 12A). 
A

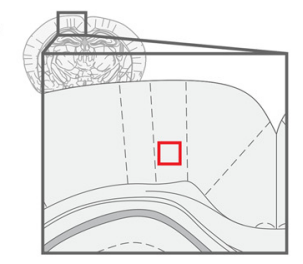

Parietal association cortex

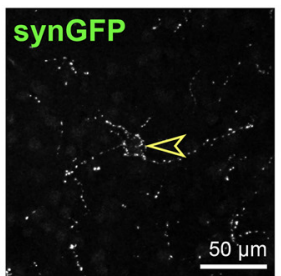

B

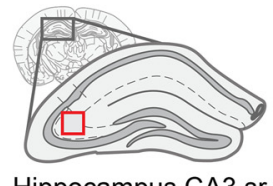

Hippocampus CA3 sr

\section{C}

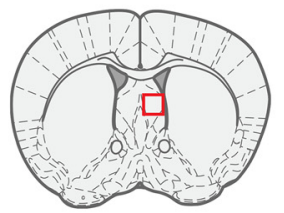
Lateral Septum
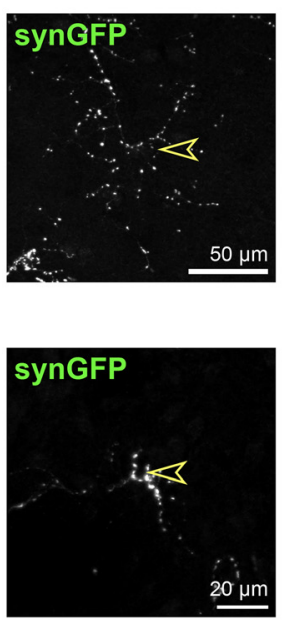

Intermediate
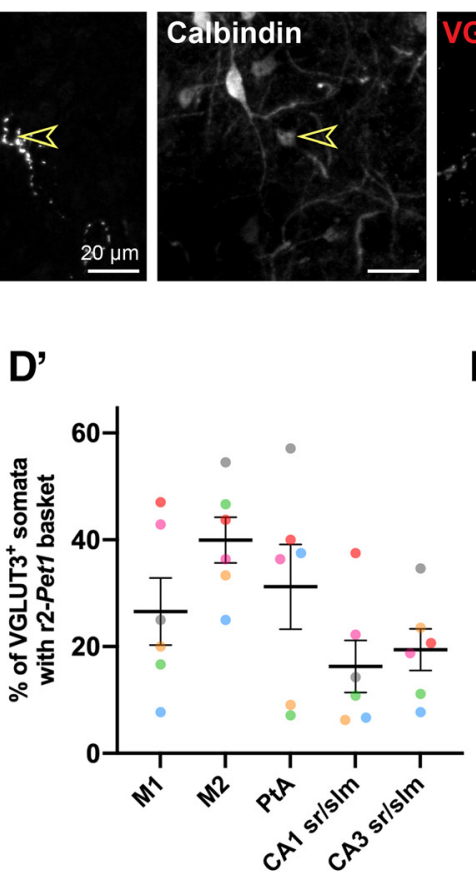

D

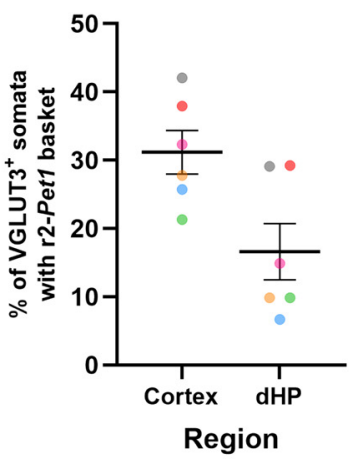

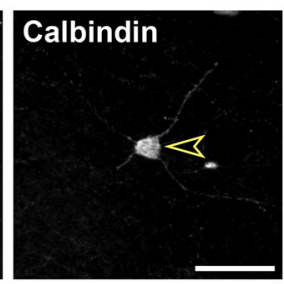
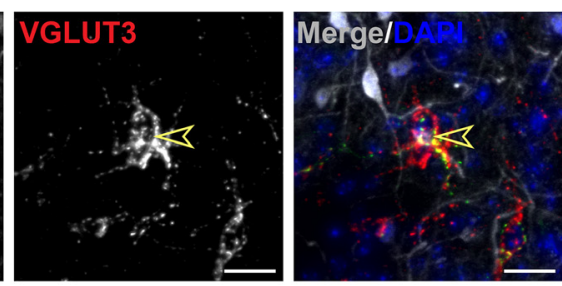

E
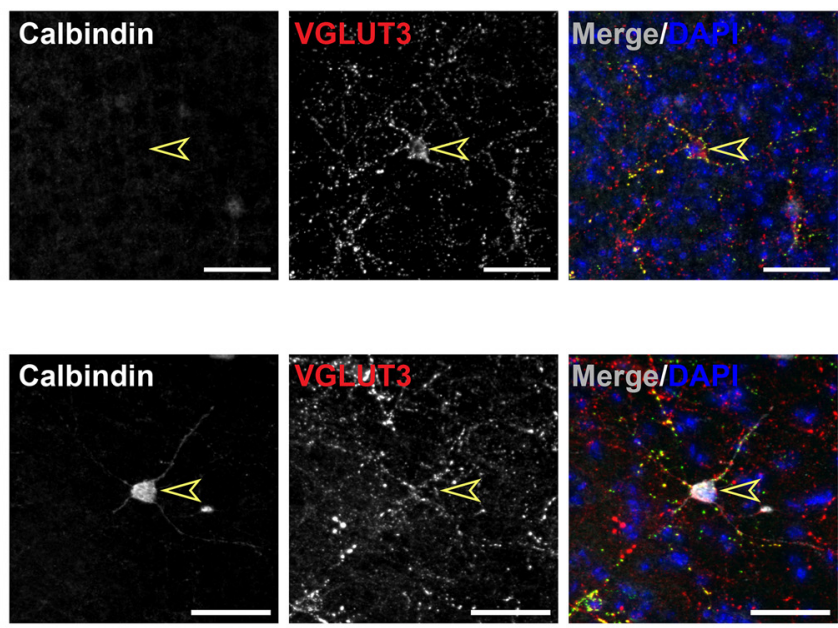

\section{Subregion}

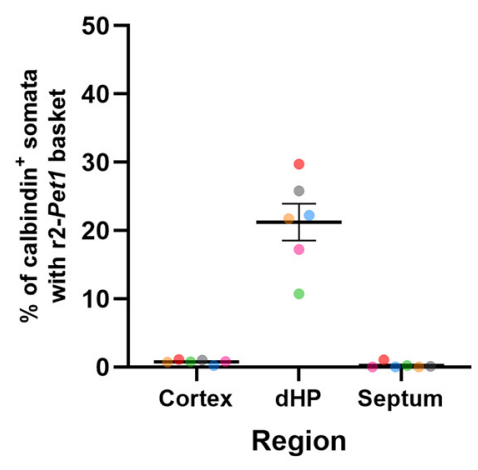

Figure 12. $\quad$ r2-Pet1 pericellular baskets exhibit regional target cell-type specificity. $\boldsymbol{A}, \boldsymbol{B}$, Examples of $\mathrm{r} 2$-Pet1 pericellular baskets targeting VGLUT3 ${ }^{+}$somata in cortex $(\boldsymbol{A})$ and calbindin ${ }^{+}$ somata in dorsal hippocampus (B). C, Rarely, calbindin ${ }^{+}$cells are targeted by r2-Pet1 baskets in the lateral septum. Arrowheads indicate the cell body targeted by the r2-Pet1 basket. $\boldsymbol{D}, \boldsymbol{D}^{\prime}$, VGLUT3 $^{+}$somata were more likely to be targeted by $\mathrm{r} 2$-Pet1 baskets in cortex than hippocampus ( $\mathrm{dHP} ; t=8.60, \mathrm{df}=5, p=0.0004$, paired $t$-test; $\boldsymbol{D}$ ), with some variation across primary motor cortex (M1), secondary motor cortex (M2), and parietal association cortex (PtA), and hippocampal subfields CA1 and CA3 sr/sIm ( $\left.\boldsymbol{D}^{\prime}\right)$. $\boldsymbol{E}$, In contrast, a higher proportion of calbindin ${ }^{+}$neurons were targeted by $\mathrm{r} 2$-Pet 1 baskets in the $\mathrm{dHP}$ than the cortex or septum $(t=7.65, \mathrm{df}=5, p=0.0006$, paired $t$ test; $\boldsymbol{E})$. Asterisks indicate statistically significant differences.

Calbindin $^{+}$somata were highly abundant in cortical layer $2 / 3$ and sporadically present in deeper layers, were distributed through the sr and sr/slm of the dorsal hippocampus (Fig. 12B), and were enriched in the lateral septum (Fig. 12C). We quantified the proportion of each cell type targeted by $\mathrm{r} 2$-Pet 1 baskets and found it varied as a function of region. We observed a higher proportion of cortical VGLUT3 ${ }^{+}$somata $(31.2 \pm 3.18 \%, 343$ total cells examined) were targeted by an $\mathrm{r} 2$-Pet 1 pericellular basket relative to hippocampal VGLUT3 ${ }^{+}$somata $(16.6 \pm 4.12 \%$; 329 cells examined; $t=8.60, \mathrm{df}=5, p=0.0004$, paired $t$ test; Fig. $12 D)$. Examining subregions of cortex and hippocampus in which we observed an average of $\geq 10$ cells per animal, we found that the greatest percentage of $\mathrm{VGLUT}^{+}{ }^{+}$somata was targeted in secondary motor cortex (M2; 39.9 $\pm 4.29 \%)$ compared with other cortical subregions [primary motor cortex (M1), $26.6 \pm 6.29$; parietal association cortex (PtA), 31.2 $\pm 7.93 \%]$ and without appreciable difference between the two hippocampal subregions analyzed (CA1 sr/slm, $16.3 \pm 4.87 \%$; CA3 sr $/ \mathrm{slm}$, 19.4 $\pm 3.90 \%$; Fig. $12 D^{\prime}$ ). Regional targeting of calbindin ${ }^{+}$interneurons exhibited the opposite pattern: cortical calbindin ${ }^{+}$neurons were rarer targets $(0.771 \pm 0.127 \%, 15,331$ total cells examined) compared with dorsal hippocampal calbindin ${ }^{+}$neurons $(21.2 \pm 2.71 \%, 225$ total cells examined; $t=7.65, \mathrm{df}=5$, $p=0.0006$, paired $t$-test; Fig. $12 E$ ). In the septum, there were no VGLUT3 $^{+}$somata, and calbindin ${ }^{+}$somata were rarely targeted by $\mathrm{r} 2$-Pet 1 baskets $(0.229 \pm 0.170 \%$ of cells targeted, 2919 total 
cells examined). These results demonstrate target specificity of r2Pet1 baskets wherein different neuron types were preferentially targeted in different regions.

\section{Discussion}

\section{Strategy}

The r2-Pet1 neuron group is hodologically, functionally, and molecularly distinct from other $\mathrm{Pet}^{+}$neurons and itself is composed of two subgroups-the r2-Pet $1^{\text {Tph2-high }}$ group being serotonergic and the r2-Pet $1^{\text {Vglut3-high }}$ subgroup being largely VGLUT3 ${ }^{+}$ and glutamatergic. We proposed that the cognate axonal boutons would likely show a matching neurochemical phenotype $\left(5-\mathrm{HT}^{+}\right.$ or $\mathrm{VGLUT}^{+}$) and thus might be used to resolve downstream circuitry specific to each of these subtypes of r2-Pet1 neurons.

\section{Main findings}

Significant findings include the following. (1) r2-Pet1 boutons were found distributed anatomically by neurochemical phenotype. $5-\mathrm{HT}^{+} / \mathrm{VGLUT3}^{-}$boutons were found in the SCN PVT, and OB. The reciprocal bouton identity of $\mathrm{VGLUT}^{+}, 5-\mathrm{HT}^{-}$ was found in the hippocampus, prelimbic cortex, and septum. Rarely observed were co-positive boutons or a mix of each single-positive bouton type, the exception being in the septum. (2) Piezo2-Pet1 neurons reside within the $\mathrm{r} 2-\mathrm{Pet} 1^{\text {Tph2-high }}$ group and exhibited axonal projections to the SCN, PVT, and OB. (3) Retrograde labeling from the hippocampus illuminated somata of r2-Pet $1^{\text {Vglut } 3 \text {-high }}$, but not r2-Pet $1^{\text {Tph2-high }}$ cells. Findings 2 and 3 substantiate the bouton-to-neuron subtype matchup of finding 1 and suggest that the r2-Pet1 neuronal group is composed of two distinct neuronal subsystems that modulate different brain regions using different neurotransmitters. They likewise suggest that the majority of $\mathrm{r} 2$-Pet $1^{\text {Vglut3-high }}$ neurons are excitatory, potentially releasing glutamate as their sole transmitter, even with the expression of Pet1 and having arisen from the same progenitor cell compartment as the classically serotonergic Pet $1^{\text {Tph2-high }}$ cells. (4) Some VGLUT3 ${ }^{+}$r2-Pet1 boutons composed pericellular baskets encasing the postsynaptic neuron soma. (5) Some basket-forming boutons in the septum were both $5-\mathrm{HT}^{+}$and VGLUT3 ${ }^{+}$, suggestive of possible cotransmission of 5-HT and glutamate (Amilhon et al., 2010). (6) The r2-Pet1 baskets often encased GABAergic interneurons of the Reelin ${ }^{+}, \mathrm{VIP}^{+}$, or VGLUT3 ${ }^{+}$ classes, in some cases as composites containing fibers from different Pet1 neuron subtypes. (7) Some basket-encased, postsynaptic targets in the septum expressed the metabotropic 5-HT2C receptor, and others in the cortex and hippocampus expressed the ionotropic 5HT3A receptor, suggesting that the basket-released 5-HT may act on distinct temporal scales in different target brain regions.

\section{Separable neuronal subsystems comprise the r2-Pet1 neuron population of the median raphe}

A simple interpretation of our $\mathrm{r} 2-\mathrm{Pet} 1$ bouton neurochemical mapping is a divergent circuitry model whereby the r2Pet $1^{\text {Tph2-high }}$ and r2-Pet $1^{\text {Vglut3-high }}$ neuron subgroups innervate different brain regions and deploy different neurotransmitters (Fig. 13). While the specific functions of each subgroup have yet to be determined, this divergent circuitry model supports certain predictions. The first is that the midline subcortical projections characterizing the $\mathrm{r} 2-\mathrm{Pet} \mathrm{1}^{\text {Tph2-high }}$ subgroup may regulate sensory modulation of circadian behavior. The SCN and PVT targets are each implicated in regulating circadian rhythm and themselves are reciprocally connected structures (Weber et al., 1998; Morin and Meyer-Bernstein, 1999). Loss of MR 5-HT input to the SCN has been shown to alter circadian rhythm and increase the sensitivity to light-induced circadian phase shifting (Bradbury et al., 1997; Morin and Meyer-Bernstein, 1999) Similarly, lesions of the PVT suggest that it too may mediate circadian rhythm shifts in response to light (Salazar-Juárez et al., 2002). Thus, r2-Pet1 $1^{\text {Tph2-high }}$ projections to these regions may suppress changes in circadian rhythm by modulating regional responsiveness to deviations from the expected pattern of illumination.

VGLUT3 $^{+}$r2-Pet 1 boutons, by contrast, were found in limbic regions such as the septum, hippocampus, and prelimbic cortex, suggesting that $\mathrm{r} 2$-Pet $1^{\text {Vglut3-high }}$ neurons may modulate memory and sensorimotor gating. The medial septum and hippocampus are involved in generating theta rhythm, an oscillating pattern of brain activity that contributes to spatial and episodic memory formation (Buzsáki, 2002; Hasselmo, 2005; Munn et al., 2015). Stimulation of the MR has been shown to desynchronize theta (Vertes, 1981; Vinogradova et al., 1999; Jackson et al., 2008; Wang et al., 2015). Though this effect has previously been attributed to 5-HT release (Assaf and Miller, 1978; Kinney et al., 1996), recent work has suggested there also exists a nonserotonergic mechanism (Jackson et al., 2008). If r2-Pet1 ${ }^{\text {Vglut3-high }}$ neurons desynchronize theta, their activity may perturb memory formation (Vertes, 2005). Indeed, in line with this hypothesis, recent experiments acutely inhibiting $\mathrm{r} 2$-Pet 1 neurons en masse during conditioned learning led to a later failure in the normal extinction of that conditioned place preference (Baskin et al., 2020). Finally, chronic silencing of r2-Pet1 neurons has been shown to enhance prepulse inhibition (PPI), a measure of sensorimotor gating (Okaty et al., 2015). Given the extensive VGLUT3 $^{+}$r2-Pet1 innervation of regions that modulate PPI, such as the prelimbic cortex and hippocampus (Bakshi and Geyer, 1998; Rohleder et al., 2016), and the sensitivity of VGLUT3 $^{+}$MR neurons to sensory stimulation (Domonkos et al., 2016), we suggest that this effect may have been driven by a loss of neurotransmission from the r2-Pet $1^{\text {Vglut3-high }}$ neuron subtype. Thus, we predict $\mathrm{r} 2-\mathrm{Pet} 1^{\mathrm{Vglut} 3-\mathrm{high}}$ neurons may regulate sensorimotor gating and modulate theta to affect memory.

\section{Pericellular baskets characterize some r2-Pet1 ${ }^{\text {Vglut3-high }}$ axonal collaterals}

We observed VGLUT3 ${ }^{+}$r2-Pet1 boutons in pericellular baskets in hippocampus, cortex, and septum, with baskets of the latter region also containing $5-\mathrm{HT}^{+} / \mathrm{VGLUT}^{+}$co-positive $\mathrm{r} 2$-Pet 1 boutons. We propose that these co-positive boutons derive from r2-Pet $1^{\text {Vglut3-high }}$ collaterals because our analyses of Piezo2-Pet1 fibers from the r2-Pet $1^{\text {Tph2-high }}$ subgroup failed to reveal baskets. This would further suggest that a fraction of $\mathrm{r} 2-$ Pet $1^{\text {Vglut } 3 \text {-high }}$ cells express sufficient, although low, levels of TPH2 or the 5-HT reuptake transporter SLC6A4 to generate $5-\mathrm{HT}^{+}$boutons. Pericellular baskets formed by $5-\mathrm{HT}^{+}$fibers (Köhler et al., 1982; Freund et al., 1990; Hornung et al., 1990; Dinopoulos et al., 1993; Leger et al., 2001) and VGLUT3 ${ }^{+}$fibers (Hioki et al., 2004; Riedel et al., 2008; Amilhon et al., 2010) have been reported previously; we suggest that fibers from r2-Pet1 ${ }^{\text {Vglut3-high }}$ cells may be reflected, in part, in the latter.

Also revealed here are composite baskets, where axons from multiple Pet1 neuron subtypes converge on a single targeted cell in cortex, hippocampus, and septum. In lateral septum composite baskets, r2-Pet1 varicosities were less likely to be $5-\mathrm{HT}^{+}$, while both r2-Pet 1 and other Pet 1 varicosities were similarly likely to be VGLUT3 ${ }^{+}$, suggesting that these subtypes of Pet 1 neurons may release different neurotransmitters onto the baskettargeted cell. The commonality of VGLUT3 in baskets suggests 
A

Predominant
r2-Pet1 bouton
phenotype
$\square 5$-HT
$\square$ VGLUT3+ $^{+}$
$\square$ Both $^{+}$

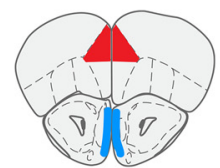

Prelimbic Cortex and Olfactory Bulb (Ctx, OB)

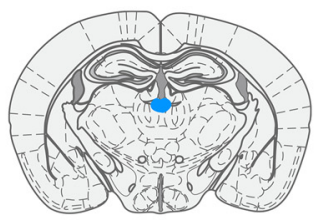

Paraventricular Thalamic Nucleus (PVT)

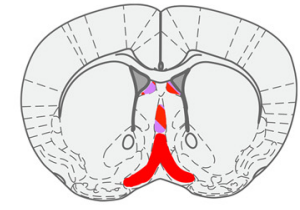

Medial and Lateral Septum (MS, LS) and Diagonal Band (DB)

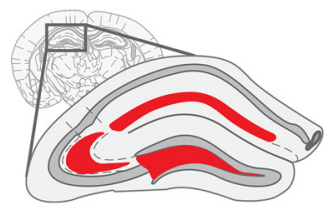

Rostral Hippocampus (HP)

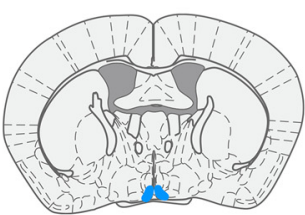

\section{Suprachiasmatic Nucleus (SCN)}

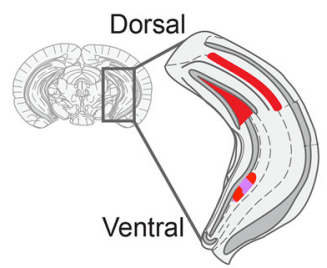

Caudal

Hippocampus (HP)
B

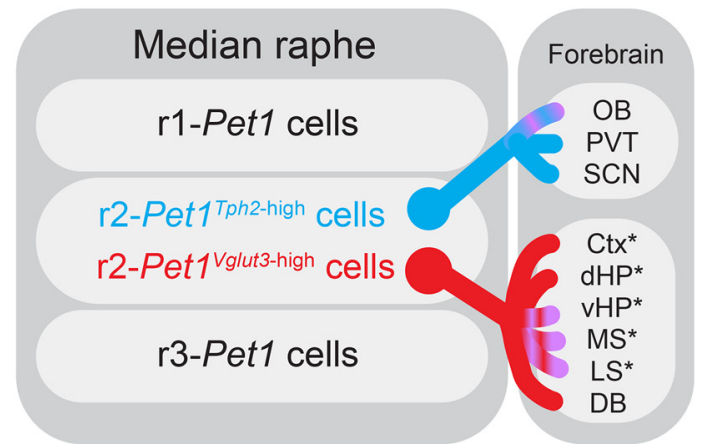

C In *-marked regions in B:

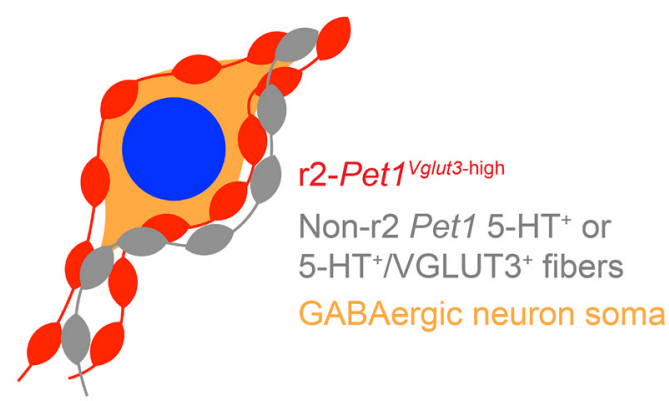

Figure 13. A, Summary of findings. r2-Pet $1 \mathrm{VGLUT}^{+}$and $5-\mathrm{HT}^{+}$boutons are spatially segregated across the forebrain, with cortical and hippocampal areas innervated by VGLUT3 ${ }^{+}$r2Pet1 boutons; midline structures such as the SCN, OB, and PVT innervated by $5-\mathrm{HT}^{+}$r2-Pet1 boutons; and septal areas innervated by a mix of VGLUT3 ${ }^{+}$and copositive 5 - $\mathrm{HT}^{+} / \mathrm{NGLUT3}^{+}$boutons. $\boldsymbol{B}$, These results suggest functionally separable subtypes of r2-Pet1 cells. $\boldsymbol{C}$, In the septum, cortex, and hippocampus, regions targeted by the r2-Pet $7^{\text {Vglut3-high }}$ subtype, r2-Pet1 boutons formed pericellular baskets targeting inhibitory interneurons, suggesting additional functional specialization of this r2-Pet1 subset.

that it may play a role in basket function, possibly by synergizing cotransmission of 5-HT and glutamate (Amilhon et al., 2010). Given that previous retrograde tracing studies identified inputs from both the DR and MR to the LS (Köhler et al., 1982; Deng et al., 2019); we suggest that the other source of non-r2 Pet1 5- $\mathrm{HT}^{+}$ input to pericellular baskets is from En1-Pet1 neurons arising embryonically from $\mathrm{r} 1$ and/or $\mathrm{r} 0$ (Jensen et al., 2008). These results suggest that pericellular baskets are complex, potentially releasing either $5-\mathrm{HT}$, glutamate, or both depending on which set of Pet 1 neurons and associated axonal terminals are excited.

In the cortex, we observed $\mathrm{r} 2-$ Pet $1 \mathrm{VGLUT3}^{+}$pericellular baskets around subtypes of GABAergic interneurons. A particularly intriguing target was a set of inhibitory neurons that themselves were also VGLUT3 ${ }^{+}$and which likely correspond to a subset of CCKexpressing interneurons referred to as "basket cells" (Somogyi et al., 2004; Fasano et al., 2017; Harris et al., 2018). Previous work proposed that the source of these presynaptic basket structures encasing these VGLUT3 ${ }^{+}$basket interneurons was intracortical rather than long-range from the raphe, because the baskets persisted after pharmacological ablation of serotonin neurons with 5,7-DHT (Hioki et al., 2004). It is possible that $\mathrm{r} 2-P e t 1^{\text {Vglut3-high }}$ neurons are poorly killed by 5,7-DHT, because of their reduced expression of serotonin transporter SLC6A4, monoamine oxidase A (MAOA), and/or
MAOB (Okaty et al., 2015), which may be important for the uptake and toxicity of 5,7-DHT (Finnegan et al., 1989; Paterak and Stefański, 2014). Additionally, there are likely multiple sources for these VGLUT3 ${ }^{+}$presynaptic baskets, such that the loss of those composed of fibers from the MR goes undetected. Our work then clarifies the existence of a long-range pericellular basket-forming projection from Pet1 MR cells to a specific subgroup of cortical interneurons.

An analogous projection from $\mathrm{r} 2-$ Pet1 neurons to VGLUT3 ${ }^{+}$ putative basket cell interneurons in the hippocampus was also observed. As in the cortex, many of the postsynaptic cells were immunopositive for 5-HT3A, the ionotropic 5-HT receptor. It has been suggested that an MR glutamatergic input to 5-HT3Aexpressing hippocampal interneurons "prepotentiates" them to be responsive to subsequent presentation of 5-HT (Jackson et al., 2009). We suggest that the r2-Pet $1^{\text {Vglut } 3 \text {-high }}$ subtype is well positioned for this role, as it targets 5-HT3A-expressing interneurons and, by forming pericellular baskets around these cells, could deliver strong, temporally precise excitation immediately before 5 -HT release. This circuit could allow r2-Pet $1^{\text {Vglut } 3 \text {-high }}$ neurons to control the activity of basket cell interneurons that then each have highly collateralized axons extending to many principal cells, giving the r2-Pet1 neuron a privileged position for tuning overall cortical and hippocampal network activity. 


\section{Conclusion}

We describe neurochemically divergent groups of r2-Pet1 neurons, one largely serotonergic and one largely glutamatergic, each arising from the same embryonic progenitor zone, rhombomere 2 . We propose that the anatomic segregation of their collective ascending boutons by neurochemical phenotype reflects two separate, largely non-overlapping neuronal subsystems that may each mediate distinct functions of the median raphe. The work described here uncovers a new level of structural organization within the median raphe.

\section{References}

Abela AR, Browne CJ, Sargin D, Prevot TD, Ji XD, Li Z, Lambe EK, Fletcher PJ (2020) Median raphe serotonin neurons promote anxiety-like behavior via inputs to the dorsal hippocampus. Neuropharmacology 168:107985.

Agnati LF, Zoli M, Strömberg I, Fuxe K (1995) Intercellular communication in the brain: wiring versus volume transmission. Neuroscience 69:711726.

Amilhon B, Lepicard E, Renoir T, Mongeau R, Popa D, Poirel O, Miot S, Gras C, Gardier AM, Gallego J, Hamon M, Lanfumey L, Gasnier B, Giros B, El Mestikawy S (2010) VGLUT3 (vesicular glutamate transporter type 3) contribution to the regulation of serotonergic transmission and anxiety. J Neurosci 30:2198-2210.

Assaf SY, Miller JJ (1978) The role of a raphe serotonin system in the control of septal unit activity and hippocampal desynchronization. Neuroscience 3:539-550.

Awatramani R, Soriano P, Rodriguez C, Mai JJ, Dymecki SM (2003) Cryptic boundaries in roof plate and choroid plexus identified by intersectional gene activation. Nat Genet 35:70-75.

Azmitia EC, Segal M (1978) An autoradiographic analysis of the differential ascending projections of the dorsal and median raphe nuclei in the rat. J Comp Neurol 179:641-667.

Bakshi VP, Geyer MA (1998) Multiple limbic regions mediate the disruption of prepulse inhibition produced in rats by the noncompetitive NMDA antagonist dizocilpine. J Neurosci 18:8394-8401.

Bang SJ, Jensen P, Dymecki SM, Commons KG (2012) Projections and interconnections of genetically defined serotonin neurons in mice. Eur J Neurosci 35:85-96.

Baskin BM, Mai JJ, Dymecki SM, Kantak KM (2020) Cocaine reward and memory after chemogenetic inhibition of distinct serotonin neuron subtypes in mice. Psychopharmacology (Berl) 237:2633-2648.

Beaudet A, Descarries L (1981) The fine structure of central serotonin neurons. J Physiol (Paris) 77:193-203.

Booker SA, Vida I (2018) Morphological diversity and connectivity of hippocampal interneurons. Cell Tissue Res 373:619-641.

Bradbury MJ, Dement WC, Edgar DM (1997) Serotonin-containing fibers in the suprachiasmatic hypothalamus attenuate light-induced phase delays in mice. Brain Res 768:125-134.

Brust RD, Corcoran AE, Richerson GB, Nattie E, Dymecki SM (2014) Functional and developmental identification of a molecular subtype of brain serotonergic neuron specialized to regulate breathing dynamics. Cell Rep 9:2152-2165.

Buzsáki G (2002) Theta oscillations in the hippocampus. Neuron 33:325340.

Chang W, Kanda H, Ikeda R, Ling J, DeBerry JJ, Gu JG (2016) Merkel disc is a serotonergic synapse in the epidermis for transmitting tactile signals in mammals. Proc Natl Acad Sci U S A 113:E5491-E5500.

DeFelipe J, Hendry SH, Hashikawa T, Jones EG (1991) Synaptic relationships of serotonin-immunoreactive terminal baskets on GABA neurons in the cat auditory cortex. Cereb Cortex 1:117-133.

De-Miguel FF, Trueta C (2005) Synaptic and extrasynaptic secretion of serotonin. Cell Mol Neurobiol 25:297-312.

Deng K, Yang L, Xie J, Tang H, Wu G-S, Luo H-R (2019) Whole-brain mapping of projection from mouse lateral septal nucleus. Biol Open 8: bio043554.

De Paola V, Arber S, Caroni P (2003) AMPA receptors regulate dynamic equilibrium of presynaptic terminals in mature hippocampal networks. Nat Neurosci 6:491-500.
Dinopoulos A, Dori I, Parnavelas JG (1993) Serotonergic innervation of the mature and developing lateral septum of the rat: a light and electron microscopic immunocytochemical analysis. Neuroscience 55:209-222.

Domonkos A, Nikitidou Ledri L, Laszlovszky T, Cserép C, Borhegyi Z, Papp E, Nyiri G, Freund TF, Varga V (2016) Divergent in vivo activity of nonserotonergic and serotonergic VGluT3-neurones in the median raphe region. J Physiol 594:3775-3790.

Dymecki SM, Ray RS, Kim JC (2010) Mapping cell fate and function using recombinase-based intersectional strategies. Methods Enzymol 477:183213.

Fasano C, Rocchetti J, Pietrajtis K, Zander J-F, Manseau F, Sakae DY, Marcus-Sells M, Ramet L, Morel LJ, Carrel D, Dumas S, Bolte S, Bernard V, Vigneault E, Goutagny R, Ahnert-Hilger G, Giros B, Daumas S, Williams S, El Mestikawy S (2017) Regulation of the hippocampal network by VGLUT3-positive CCK- GABAergic basket cells. Front Cell Neurosci 11:140.

Finnegan KT, DeLanney LE, Irwin I, Ricaurte GA, Langston JW (1989) The amine-depleting effects of 5,7-dihydroxytryptamine (5,7-DHT) in C57BL/6 mice do not increase with age. Brain Res 496:251-256.

Franklin K, Paxinos G (2008) The mouse brain in stereotaxic coordinates. Amsterdam: Academic.

Fremeau RT, Voglmaier S, Seal RP, Edwards RH (2004) VGLUTs define subsets of excitatory neurons and suggest novel roles for glutamate. Trends Neurosci 27:98-103.

Freund TF, Gulyás AI, Acsády L, Görcs T, Tóth K (1990) Serotonergic control of the hippocampus via local inhibitory interneurons. Proc Natl Acad Sci U S A 87:8501-8505.

Gaspar P, Lillesaar C (2012) Probing the diversity of serotonin neurons. Philos Trans R Soc Lond B Biol Sci 367:2382-2394.

Glass JD, Grossman GH, Farnbauch L, DiNardo L (2003) Midbrain raphe modulation of nonphotic circadian clock resetting and 5-HT release in the mammalian suprachiasmatic nucleus. J Neurosci 23:7451-7460.

Halasy K, Miettinen R, Szabat E, Freund TF (1992) GABAergic interneurons are the major postsynaptic targets of median raphe afferents in the rat dentate gyrus. Eur J Neurosci 4:144-153.

Harris KD, Hochgerner H, Skene NG, Magno L, Katona L, Gonzales CB, Somogyi P, Kessaris N, Linnarsson S, Hjerling-Leffler J (2018) Classes and continua of hippocampal CA1 inhibitory neurons revealed by singlecell transcriptomics. PLoS Biol 16:e2006387.

Hasselmo ME (2005) What is the function of hippocampal theta rhythm?Linking behavioral data to phasic properties of field potential and unit recording data. Hippocampus 15:936-949.

Herzog E, Gilchrist J, Gras C, Muzerelle A, Ravassard P, Giros B, Gaspar P, El Mestikawy S (2004) Localization of VGLUT3, the vesicular glutamate transporter type 3, in the rat brain. Neuroscience 123:983-1002.

Hioki H, Fujiyama F, Nakamura K, Wu S-X, Matsuda W, Kaneko T (2004) Chemically specific circuit composed of vesicular glutamate transporter 3 - and preprotachykinin B-producing interneurons in the rat neocortex. Cereb Cortex 14:1266-1275.

Hornung JP, Celio MR (1992) The selective innervation by serotoninergic axons of calbindin-containing interneurons in the neocortex and hippocampus of the marmoset. J Comp Neurol 320:457-467.

Hornung JP, Fritschy JM, Törk I (1990) Distribution of two morphologically distinct subsets of serotoninergic axons in the cerebral cortex of the marmoset. J Comp Neurol 297:165-181.

Iwasaki K, Komiya H, Kakizaki M, Miyoshi C, Abe M, Sakimura K, Funato H, Yanagisawa M (2018) Ablation of central serotonergic neurons decreased REM sleep and attenuated arousal response. Front Neurosci 12:535.

Jackson J, Dickson CT, Bland BH (2008) Median raphe stimulation disrupts hippocampal theta via rapid inhibition and state-dependent phase reset of theta-related neural circuitry. J Neurophysiol 99:3009-3026.

Jackson J, Bland BH, Antle MC (2009) Nonserotonergic projection neurons in the midbrain raphe nuclei contain the vesicular glutamate transporter VGLUT3. Synapse 63:31-41.

Jensen P, Farago AF, Awatramani RB, Scott MM, Deneris ES, Dymecki SM (2008) Redefining the serotonergic system by genetic lineage. Nat Neurosci 11:417-419.

Kelsch W, Lin C-W, Lois C (2008) Sequential development of synapses in dendritic domains during adult neurogenesis. Proc Natl Acad Sci U S A 105:16803-16808. 
Kinney GG, Kocsis B, Vertes RP (1996) Medial septal unit firing characteristics following injections of 8-OH-DPAT into the median raphe nucleus. Brain Res 708:116-122.

Kiyasova V, Gaspar P (2011) Development of raphe serotonin neurons from specification to guidance. Eur J Neurosci 34:1553-1562.

Köhler C, Chan-Palay V, Steinbusch H (1982) The distribution and origin of serotonin-containing fibers in the septal area: a combined immunohistochemical and fluorescent retrograde tracing study in the rat. J Comp Neurol 209:91-111.

Kosofsky BE, Molliver ME (1987) The serotoninergic innervation of cerebral cortex: different classes of axon terminals arise from dorsal and median raphe nuclei. Synapse 1:153-168.

Lee S, Hjerling-Leffler J, Zagha E, Fishell G, Rudy B (2010) The largest group of superficial neocortical GABAergic interneurons expresses ionotropic serotonin receptors. J Neurosci 30:16796-16808.

Leger L, Charnay Y, Hof PR, Bouras C, Cespuglio R (2001) Anatomical distribution of serotonin-containing neurons and axons in the central nervous system of the cat. J Comp Neurol 433:157-182.

Li L, Tasic B, Micheva KD, Ivanov VM, Spletter ML, Smith SJ, Luo L (2010) Visualizing the distribution of synapses from individual neurons in the mouse brain. PLoS One 5:e11503.

Maddaloni G, Bertero A, Pratelli M, Barsotti N, Boonstra A, Giorgi A, Migliarini S, Pasqualetti M (2017) Development of serotonergic fibers in the post-natal mouse brain. Front Cell Neurosci 11:202.

Madisen L, Mao T, Koch H, Zhuo J-m, Berenyi A, Fujisawa S, Hsu Y-WA, Garcia AJ, Gu X, Zanella S, Kidney J, Gu H, Mao Y, Hooks BM, Boyden ES, Buzsáki G, Ramirez JM, Jones AR, Svoboda K, Han X, et al. (2012) A toolbox of Cre-dependent optogenetic transgenic mice for light-induced activation and silencing. Nat Neurosci 15:793-802.

Madisen L, Garner AR, Shimaoka D, Chuong AS, Klapoetke NC, Li L, van der Bourg A, Niino Y, Egolf L, Monetti C, Gu H, Mills M, Cheng A, Tasic B, Nguyen TN, Sunkin SM, Benucci A, Nagy A, Miyawaki A, Helmchen F, et al. (2015) Transgenic mice for intersectional targeting of neural sensors and effectors with high specificity and performance. Neuron 85:942-958.

Meyer-Bernstein L, Morin P (1996) Differential serotonergic innervation of the suprachiasmatic nucleus and the intergeniculate leaflet and its role in circadian rhythm modulation. J Neurosci 16:2097-2111.

Morin LP, Meyer-Bernstein EL (1999) The ascending serotonergic system in the hamster: comparison with projections of the dorsal and median raphe nuclei. Neuroscience 91:81-105.

Munn RGK, Tyree SM, McNaughton N, Bilkey DK (2015) The frequency of hippocampal theta rhythm is modulated on a circadian period and is entrained by food availability. Front Behav Neurosci 9:61.

Niederkofler V, Asher TE, Okaty BW, Rood BD, Narayan A, Hwa LS, Beck SG, Miczek KA, Dymecki SM (2016) Identification of serotonergic neuronal modules that affect aggressive behavior. Cell Rep 17:1934-1949.

Ohmura Y, Izumi T, Yamaguchi T, Tsutsui-Kimura I, Yoshida T, Yoshioka $M$ (2010) The serotonergic projection from the median raphe nucleus to the ventral hippocampus is involved in the retrieval of fear memory through the corticotropin-releasing factor type 2 receptor. Neuropsychopharmacology 35:1271-1278.

Okaty BW, Freret ME, Rood BD, Brust RD, Hennessy ML, deBairos D, Kim JC, Cook MN, Dymecki SM (2015) Multi-scale molecular deconstruction of the serotonin neuron system. Neuron 88:774-791.

Okaty BW, Commons KG, Dymecki SM (2019) Embracing diversity in the 5-HT neuronal system. Nat Rev Neurosci 20:397-424.

Okaty BW, Sturrock N, Escobedo Lozoya Y, Chang Y, Senft RA, Lyon KA, Alekseyenko OV, Dymecki SM (2020) A single-cell transcriptomic and anatomic atlas of mouse dorsal raphe Pet1 neurons. ELife 9:e55523.

Papp EC, Hájos N, Acsády L, Freund TF (1999) Medial septal and median raphe innervation of vasoactive intestinal polypeptide-containing interneurons in the hippocampus. Neuroscience 90:369-382.

Paterak J, Stefański R (2014) 5,6- and 5,7-Dihydroxytryptamines as Serotoninergic Neurotoxins. In: Handbook of neurotoxicity (Kostrzewa RM, ed), pp 299-325. New York: Springer.

Pelosi B, Migliarini S, Pacini G, Pratelli M, Pasqualetti M (2014) Generation of Pet1210-Cre transgenic mouse line reveals non-serotonergic expression domains of Pet1 both in CNS and periphery. PLoS One 9:e104318.
Plummer NW, Evsyukova IY, Robertson SD, de Marchena J, Tucker CJ, Jensen P (2015) Expanding the power of recombinase-based labeling to uncover cellular diversity. Development 142:4385-4393.

Ren J, Isakova A, Friedmann D, Zeng J, Grutzner SM, Pun A, Zhao GQ, Kolluru SS, Wang R, Lin R, Li P, Li A, Raymond JL, Luo Q, Luo M, Quake SR, Luo L (2019) Single-cell transcriptomes and whole-brain projections of serotonin neurons in the mouse dorsal and median raphe nuclei. Elife 8:e49424.

Riedel A, Westerholz S, Braun K, Edwards RH, Arendt T, Härtig W (2008) Vesicular glutamate transporter 3-immunoreactive pericellular baskets ensheath a distinct population of neurons in the lateral septum. J Chem Neuroanat 36:177-190.

Rohleder C, Wiedermann D, Neumaier B, Drzezga A, Timmermann L, Graf R, Leweke FM, Endepols H (2016) The functional networks of prepulse inhibition: neuronal connectivity analysis based on FDG-PET in awake and unrestrained rats. Front Behav Neurosci 10:148.

Salazar-Juárez A, Escobar C, Aguilar-Roblero R (2002) Anterior paraventricular thalamus modulates light-induced phase shifts in circadian rhythmicity in rats. Am J Physiol Regul Integr Comp Physiol 283:R897-R904.

Schallier A, Smolders I, Van Dam D, Loyens E, De Deyn PP, Michotte A, Michotte Y, Massie A (2011) Region- and age-specific changes in glutamate transport in the A $\beta$ PP23 mouse model for Alzheimer's disease. J Alzheimers Dis 24:287-300.

Schindelin J, Arganda-Carreras I, Frise E, Kaynig V, Longair M, Pietzsch T, Preibisch S, Rueden C, Saalfeld S, Schmid B, Tinevez J-Y, White DJ, Hartenstein V, Eliceiri K, Tomancak P, Cardona A (2012) Fiji: an opensource platform for biological-image analysis. Nat Methods 9:676-682.

Schneider CA, Rasband WS, Eliceiri KW (2012) NIH Image to ImageJ: 25 years of image analysis. Nat Methods 9:671-675.

Somogyi J, Baude A, Omori Y, Shimizu H, Mestikawy SE, Fukaya M, Shigemoto R, Watanabe M, Somogyi P (2004) GABAergic basket cells expressing cholecystokinin contain vesicular glutamate transporter type 3 (VGLUT3) in their synaptic terminals in hippocampus and isocortex of the rat. Eur J Neurosci 19:552-569.

Sos KE, Mayer MI, Cserép C, Takács FS, Szónyi A, Freund TF, Nyiri G (2017) Cellular architecture and transmitter phenotypes of neurons of the mouse median raphe region. Brain Struct Funct 222:287-299.

Szőnyi A, Mayer MI, Cserép C, Takács VT, Watanabe M, Freund TF, Nyiri $\mathrm{G}$ (2016) The ascending median raphe projections are mainly glutamatergic in the mouse forebrain. Brain Struct Funct 221:735-751.

Teissier A, Chemiakine A, Inbar B, Bagchi S, Ray RS, Palmiter RD, Dymecki SM, Moore H, Ansorge MS (2015) Activity of Raphé serotonergic neurons controls emotional behaviors. Cell Rep 13:1965-1976.

Tripodi M, Stepien AE, Arber S (2011) Motor antagonism exposed by spatial segregation and timing of neurogenesis. Nature 479:61-66.

Trueta C, De-Miguel FF (2012) Extrasynaptic exocytosis and its mechanisms: a source of molecules mediating volume transmission in the nervous system. Front Physiol 3:319.

Vertes RP (1981) An analysis of ascending brain stem systems involved in hippocampal synchronization and desynchronization. J Neurophysiol 46:1140-1159.

Vertes RP (2005) Hippocampal theta rhythm: a tag for short-term memory. Hippocampus 15:923-935.

Vinogradova OS, Kitchigina VF, Kudina TA, Zenchenko KI (1999) Spontaneous activity and sensory responses of hippocampal neurons during persistent theta-rhythm evoked by median raphe nucleus blockade in rabbit. Neuroscience 94:745-753.

Vu DH, Törk I (1992) Differential development of the dual serotoninergic fiber system in the cerebral cortex of the cat. J Comp Neurol 317:156-174.

Wang DV, Yau H-J, Broker CJ, Tsou J-H, Bonci A, Ikemoto S (2015) Mesopontine median raphe regulates hippocampal ripple oscillation and memory consolidation. Nat Neurosci 18:728-735.

Wang F, Flanagan J, Su N, Wang L-C, Bui S, Nielson A, Wu X, Vo H-T, Ma X-J, Luo Y (2012) RNAscope: a novel in situ RNA analysis platform for formalin-fixed, paraffin-embedded tissues. J Mol Diagn 14:22-29.

Weber ET, Gannon RL, Rea MA (1998) Local administration of serotonin agonists blocks light-induced phase advances of the circadian activity rhythm in the hamster. J Biol Rhythms 13:209-218.

Woo S-H, Ranade S, Weyer AD, Dubin AE, Baba Y, Qiu Z, Petrus M, Miyamoto T, Reddy K, Lumpkin EA, Stucky CL, Patapoutian A (2014) Piezo2 is required for Merkel-cell mechanotransduction. Nature 509:622-626. 